\title{
Interactions between quantum probability and operator space theory
}

\author{
Quanhua Xu
}

\section{Introduction}

It is well-known that probabilistic methods are important methods in Banach space theory. As operator spaces are quantized Banach spaces, one would naturally expect that quantum probability should play a non negligible role in the young operator space theory. This is indeed the case. In fact, quantum probability and operator space theory are intimately related and there exist many interactions between them. For instance, the recent remarkable development of noncommutative martingale inequalities is directly influenced and motivated by operator space theory. In particular, the establishment of the noncommutative Doob maximal inequality by Junge [J1] is inspired by Pisier's theory of vector-valued noncommutative $L_{p}$-spaces. On the other hand, the noncommutative Burkholder/Rosenthal inequalities can be used to determine the linear structure of symmetric subspaces of noncommutative $L_{p}$-spaces (see [JX]). The recent works of Pisier/Shlyakhtenko [PS] on the operator space Grothendieck inequality and of Junge [J2] on the complete embedding of $O H$ into a noncommutative $L_{1}$ are two more beautiful examples of illustration of these interactions. Certain Khintchine type inequalities are key ingredients in both works.

It is clear today that quantum probability is of increasing importance in operator space theory. We will try to convince the reader of this in this course by presenting a very brief aspect of interactions between the two theories. Our presentation is around Khintchine type inequalities. In consequence, noncommutative $L_{p}$-spaces are at the heart of these lectures.

This course can be divided into three parts. The first one gives a brief introduction to operator space theory. We start with a short discussion on completely positive maps between $\mathrm{C}^{*}$-algebras in order to prepare the introduction to operator spaces and completely bounded maps. Our introduction to operator spaces begins with concrete operator spaces, i.e., those which are closed subspaces of $B(H)$ and the Haagerup-Paulsen-Wittstock factorization theorem for completely bounded maps, which should be understood together with its predecessor, Stinespring's factorization theorem for completely positive maps. We then pass to Ruan's fundamental characterization of abstract operator spaces. It is Ruan's theorem that allows us to do basic operations on operator spaces such that duality, quotient and interpolation. Meanwhile, some important examples of operator spaces are given, including column space $C$, row space $R$ and Pisier's operator Hilbert space $O H$. This first part ends with an outline of Pisier's vector-valued noncommutative $L_{p}$-spaces. We concentrate here only on Schatten classes.

The second part is of quantum probabilistic character. The main object of this part is various noncommutative Khintchine inequalities. It becomes clear nowadays that Khintchine type inequalities are of paramount importance in operator space theory and more generally in noncommutative analysis. The inequalities we present are those for Rademacher variables, free group generators,

Laboratoire de Mathématiques, Université de France-Comté, 25030 Besançon Cedex, France qxu@univ-fcomte.fr

Lectures given in the School Quantum potential theory: Structure and applications to physics at Greifswald, February 26 - March 10, 2007 
Voiculescu's semicircular systems and Shlyakhtenko's generalized circular systems. The proofs of these inequalities in noncommutative $L_{p}$-spaces are often quite technical and tricky. But what will be needed in the third part concerns only the case $p=1$, which is often easier. We should point out that the von Neumann algebra generated by a generalized circular system is of type III, which turns our presentation of noncommutative $L_{p}$-spaces somehow more complicated. This is, unfortunately, unavoidable in view of the complete embedding of $O H$ into a noncommutative $L_{1}$ presented later.

The short third part is devoted to Junge's complete embedding of $O H$ into a noncommutative $L_{1}$. We first present $O H$ as a quotient of a subspace of $C \oplus R$ (a theorem of Pisier), which is given by the graph of a closed densely defined operator on $\ell_{2}$. We then prove that any such graph embeds completely isomorphically into a noncommutative $L_{1}$-space.

\section{Completely positive maps}

This section gives a brief discussion on completely positive maps between $\mathrm{C}^{*}$-algebras. Our references for operator algebras are $[\mathrm{KR}]$ and $[\mathrm{T}]$. Let us fix some notations used throughout this course:

- $B(H)$ denotes the space of all bounded linear operators on a complex Hilbert space $H$.

- $A$ often denotes a $\mathrm{C}^{*}$-algebra; so $A$ can be regarded as a $\mathrm{C}^{*}$-subalgebra of $B(H)$ for some $H$. $A_{+}$denotes the positive cone of $A$.

- $M$ denotes a von Neumann algebra and $M_{*}$ the predual of $M$.

- $\mathbb{M}_{n}$ denotes the algebra of complex $n \times n$ matrices; so $\mathbb{M}_{n}$ can be identified with $B\left(\ell_{2}^{n}\right)$.

- $\mathbb{M}_{n}(A)$ denotes the algebra of $n \times n$ matrices with entries in $A$. This is again a $\mathrm{C}^{*}$-algebra. If $A \subset B(H)$, then $\mathbb{M}_{n}(A)$ is a $\mathrm{C}^{*}$-subalgebra of $\mathbb{M}_{n}(B(H)) \simeq B\left(\ell_{2}^{n}(H)\right)$.

- $\ell_{p}$ denotes the $\ell_{p}$-space of complex sequences $\left(x_{k}\right)$ such that $\left(\sum_{k}\left|x_{k}\right|^{p}\right)^{1 / p}<\infty, 1 \leq p \leq \infty$ (with the usual modification for $p=\infty$ ). The $n$-dimensional version of $\ell_{p}$ is denoted by $\ell_{p}^{n}$.

Definition 1.1 Let $u: A \rightarrow B$ be a linear map between two $\mathrm{C}^{*}$-algebras.

i) $u$ is called positive if $u\left(A_{+}\right) \subset B_{+}$.

ii) $u$ is called completely positive (c.p. for short) if $u_{n}$ is positive for every $n \geq 1$, where $u_{n}=$ $\operatorname{id}_{\mathbb{M}_{n}} \otimes u: \mathbb{M}_{n}(A) \rightarrow \mathbb{M}_{n}(B)$ is defined by $u_{n}\left(\left(x_{i j}\right)\right)=\left(u\left(x_{i j}\right)\right)$.

Remark 1.2 It is easy to check that a positive map $u: A \rightarrow B$ is automatically continuous. If in addition $A$ is unital, then $\|u\|=\|u(1)\|$ (see $[\mathrm{Pa}]$ ).

\section{Examples:}

1) Homomorphisms. Every homomorphism $\pi: A \rightarrow B$ is c.p.. By homomorphism we mean a linear map satisfying: $\pi(x y)=\pi(x) \pi(y)$ and $\pi\left(x^{*}\right)=\pi(x)^{*}$ for all $x, y \in A$. Recall that a homomorphism $\pi$ is necessarily contractive, i.e., $\|u\| \leq 1$. If in addition $\pi$ is injective, then $\pi$ is isometric.

2) Multiplications. Let $a \in A$ and define $C_{a}: A \rightarrow A$ by $C_{a}(x)=a^{*} x a$. Then $C_{a}$ is c.p. and $\left\|C_{a}\right\|=\left\|a^{*} a\right\|=\|a\|^{2}$. More generally, if $H$ and $K$ are two Hilbert spaces and $a: K \rightarrow H$ a bounded operator, then $C_{a}: B(H) \rightarrow B(K)$ defined by $C_{a}(x)=a^{*} x a$, is c.p..

The classical theorem of Stinespring states that any c.p. map is the composition of two maps of the previous types. 


\section{Theorem 1.3 (Stinespring's factorization)}

Let $A$ be a $C^{*}$-algebra and $B$ a $C^{*}$-subalgebra of $B(K)$. Let $u: A \rightarrow B$ be c.p.. Then there are a Hilbert space $H$, a representation $\pi: A \rightarrow B(H)$ (i.e., a homomorphism) and a bounded operator $a: H \rightarrow K$ such that

$$
u(x)=a^{*} \pi(x) a, \quad \forall x \in A .
$$

Namely, $u=C_{a} \circ \pi$

We refer to $[\mathrm{T}]$ for the proof of this theorem. We deduce immediately the following

Corollary 1.4 If $u: A \rightarrow B$ is c.p., then

$$
\|u\|_{c b} \stackrel{\text { def }}{=} \sup _{n}\left\|u_{n}: \mathbb{M}_{n}(A) \rightarrow \mathbb{M}_{n}(B)\right\|<\infty .
$$

Namely, $u$ is completely bounded. Moreover, $\|u\|_{c b}=\|u\|$.

\section{Exercices:}

1) Prove that a positive map is automatically continuous.

2) Let $A$ be a $C^{*}$-algebra. Prove that $x=\left(x_{i j}\right)_{i, j} \in \mathbb{M}_{n}(A)$ is positive iff $x$ is a sum of matrices of the form $\left(a_{i}^{*} a_{j}\right)_{i, j}$ with $a_{1}, \ldots, a_{n} \in A$.

3) Let $A$ and $B$ be $\mathrm{C}^{*}$-algebras with $B$ commutative. Prove that every positive map $\varphi: A \rightarrow B$ is automatically c.p..

\section{Concrete operator spaces and completely bounded maps}

We consider in this section closed subspaces of $B(H)$ and completely bounded maps between them. The analogue for the present setting of the Stinespring factorization given in the previous section is the Haagerup-Paulsen-Wittstock factorization for completely bounded maps, which is fundamental in the theory. The references for this and next sections are [ER], [Pa] and [P3].

Definition 2.1 A (concrete) operator space is a closed subspace $E$ of $B(H)$ for some Hilbert space $H$.

Let $E$ be a Banach space, and let $B_{E^{*}}$ denote the unit ball of the dual $E^{*}$ of $E$. $B_{E^{*}}$ becomes a compact topological space when equipped with the w*-topology. Then $E \subset C\left(B_{E^{*}}\right)$ isometrically. More precisely, given $x \in E$ define $\widehat{x}: B_{E^{*}} \rightarrow \mathbb{C}$ by $\widehat{x}(\xi)=\xi(x)$. Then $x \mapsto \widehat{x}$ establishes an isometry from $E$ into $C\left(B_{E^{*}}\right)$. But now $C\left(B_{E^{*}}\right)$ is a commutative $\mathrm{C}^{*}$-algebra, so $C\left(B_{E^{*}}\right) \subset B(H)$ for some $H$. In this way, any Banach space is an operator space. However, according to the preceding definition, an operator space $E$ is given together with an embedding of $E$ into a $B(H)$. More precisely, an operator space is a pair of a Banach space $E$ and an embedding of $E$ into some $B(H)$. On the other hand, $B(H)$ admits a natural matricial structure: $\mathbb{M}_{n}(B(H))=B\left(\ell_{2}^{n}(H)\right)$ for every $n \in \mathbb{N}$. This should be reflected in $E$ too. In particularly, the "admissible" morphisms in the category of operator spaces should respect this matricial structure.

Matricial structure. Let $E \subset B(H)$ be an operator space. Then $E$ inherits the matricial structure of $B(H)$ by virtue of the embedding $\mathbb{M}_{n}(E) \subset \mathbb{M}_{n}(B(H))$. More precisely, let $\mathbb{M}_{n}(E)$ be the space of $n \times n$ matrices with entries in $E$. Then $\mathbb{M}_{n}(E)$ is equipped with the norm induced by that of $B\left(\ell_{2}^{n}(H)\right)$.

Definition 2.2 Let $E \subset B(H)$ and $F \subset B(K)$ be two operator spaces. Let $u: E \rightarrow F$ be a linear map. 
i) $u$ is called completely bounded (c.b. for short) if

$$
\|u\|_{c b}=\sup _{n \geq 1}\left\|u_{n}\right\|<\infty
$$

where $u_{n}=\mathrm{id}_{\mathbb{M}_{n}} \otimes u: \mathbb{M}_{n}(E) \rightarrow \mathbb{M}_{n}(F)$ is defined by $u_{n}\left(\left(x_{i j}\right)\right)=\left(u\left(x_{i j}\right)\right)$. $C B(E, F)$ denotes the space of all c.b. maps from $E$ to $F$.

ii) $u$ is called a complete isomorphism if $u$ is a c.b. bijection and $u^{-1}$ is also c.b..

iii) $u$ is called completely isometric if $u_{n}$ is isometric for every $n$. If $u$ is a bijection and both $u$ and $u^{-1}$ are completely isometric, $u$ is called a complete isometry.

\section{Examples:}

1) $\mathbf{C}^{*}$-algebras. Let $A$ be a $\mathrm{C}^{*}$-algebra. Then $A$ is a $\mathrm{C}^{*}$-subalgebra of some $B(H)$; so $A$ is an operator space. The resulting matricial structure on $A$ is called the natural operator structure of $A$. In particular, any von Neumann algebra has its natural operator structure. Note that this natural operator space structure of $A$ is independent, up to complete isometry, of particular representation of $A$ as $\mathrm{C}^{*}$-subalgebra of $B(H)$ for a faithful representation is complete isometric.

2) Minimal structure. Let $E$ be a Banach space. Then we have the isometric embedding $E \subset C\left(B_{E^{*}}\right)$, which turns $E$ an operator space, denoted by $\min (E)$. This operator space structure is called the minimal structure on $E$. The adjective "minimal" means that this structure induces the least matricial norms on $\mathbb{M}_{n}(E)$. Indeed, assume that $E$ itself is an operator space and consider also the associated minimal operator space $\min (E)$. Then by Proposition 2.3 below, the identity map on $E$ induces a complete contraction from $E$ to $\min (E)$. Namely, for any $n$ and any $x \in \mathbb{M}_{n}(E)$

$$
\|x\|_{\mathbb{M}_{n}(\min (E))} \leq\|x\|_{\mathbb{M}_{n}(E)} .
$$

3) Maximal structure. Similarly, we can introduce a maximal operator structure on a Banach space $E$. Let $\Phi$ be the family of all pairs $\varphi=\left(u_{\varphi}, H_{\varphi}\right)$ with $H_{\varphi}$ a Hilbert space and $u_{\varphi}: E \rightarrow$ $B\left(H_{\varphi}\right)$ a contraction. Let $\mathcal{H}$ be the Hilbert space direct sum of the $H_{\varphi}$ :

$$
\mathcal{H}=\bigoplus_{\varphi \in \Phi} H_{\varphi}
$$

Then $B(\mathcal{H})$ is the $\mathrm{C}^{*}$-algebra direct sum of the $B\left(H_{\varphi}\right)$ :

$$
B(\mathcal{H})=\bigoplus_{\varphi \in \Phi} B\left(H_{\varphi}\right)
$$

Define $J: E \rightarrow B(\mathcal{H})$ by $J(x)=\left(u_{\varphi}(x)\right)_{\varphi \in \Phi}$. It is clear that $J$ is isometric. This yields an isometric embedding of $E$ into $B(\mathcal{H})$. Identifying $E$ and $J(E) \subset B(\mathcal{H})$, we turn $E$ an operator space. This operator space structure is called the maximal structure of $E$ and denoted by $\max (E)$. By definition, one sees that if $E$ itself is an operator space, then the identity map $\operatorname{id}_{E}: \max (E) \rightarrow E$ is completely contractive.

4) Column and row spaces. Let $\left(e_{i j}\right)$ denote the standard matrix units of $B\left(\ell_{2}\right)$. Define

$$
C=\overline{\operatorname{span}}\left(e_{i 1}, i \geq 1\right) \quad \text { and } \quad R=\overline{\operatorname{span}}\left(e_{1 j}, j \geq 1\right) .
$$

Thus we get two operator spaces $C \subset B\left(\ell_{2}\right)$ and $R \subset B\left(\ell_{2}\right)$. Note that $C$ (resp. $R$ ) is identified as the first column (resp. row) subspace of $B\left(\ell_{2}\right)$. The operator space structures of $C$ and $R$ are easily determined. Let $x \in \mathbb{M}_{n}(C), y \in \mathbb{M}_{n}(R)$ and write

$$
x=\sum_{i} x_{i} \otimes e_{i 1}, \quad y=\sum_{i} y_{i} \otimes e_{1 i}, \quad x_{i}, y_{i} \in \mathbb{M}_{n} .
$$


Then

$$
\|x\|_{\mathbb{M}_{n}(C)}=\left\|\sum_{i} x_{i}^{*} x_{i}\right\|^{1 / 2}, \quad\|y\|_{\mathbb{M}_{n}(R)}=\left\|\sum_{i} y_{i} y_{i}^{*}\right\|^{1 / 2} .
$$

The $n$-dimensional versions of $C$ and $R$ are denoted by $C^{n}$ and $R^{n}$, respectively. As Banach spaces both $C$ and $R$ are isometric to $\ell_{2}$ via the identification $e_{i 1} \sim e_{1 i} \sim e_{i}$, where $\left(e_{i}\right)$ denotes the canonical basis of $\ell_{2}$. We will see at the end of this section that they are not completely isomorphic.

Proposition 2.3 Let $E \subset B(H)$ be an operator space and $A$ a commutative $C^{*}$-algebra. Then any bounded map $u: E \rightarrow A$ is automatically c.b. and $\|u\|_{c b}=\|u\|$.

Proof. Let us first consider the case where $A=\mathbb{C}$. Let $u: E \rightarrow \mathbb{C}$ be a continuous linear functional with $\|u\| \leq 1$. For any $n \in \mathbb{N}$ we must show that $u_{n}: \mathbb{M}_{n}(E) \rightarrow \mathbb{M}_{n}$ is a contraction. To this end let $x \in \mathbb{M}_{n}(E)$, and let $\alpha, \beta \in \ell_{2}^{n}$ ( $\alpha$ and $\beta$ being viewed as column matrices). Then

$$
\begin{aligned}
\left|\left\langle u_{n}(x) \alpha, \beta\right\rangle\right| & =\left|\sum_{i, j=1}^{n} u\left(x_{i j}\right) \alpha_{j} \bar{\beta}_{i}\right|=\left|u\left(\sum_{i, j=1}^{n} x_{i j} \alpha_{j} \bar{\beta}_{i}\right)\right| \\
& \leq\left\|\sum_{i, j=1}^{n} x_{i j} \alpha_{j} \bar{\beta}_{i}\right\|=\left\|\beta^{*} x \alpha\right\| \leq\|\beta\|\|x\|\|\alpha\| .
\end{aligned}
$$

Taking the supremum over all $x, \alpha, \beta$ in the respective unit balls, we deduce that $\left\|u_{n}\right\| \leq 1$. Thus $u$ is a complete contraction.

The general case can be easily reduced to the previous one. Indeed, since $A$ is commutative, we can assume $A=C_{0}(\Omega)$ for some locally compact topological space $\Omega$, where $C_{0}(\Omega)$ denotes the $\mathrm{C}^{*}$ algebra of all continuous functions on $\Omega$ which tend to zero at infinity. Then $\mathbb{M}_{n}(A)=C_{0}\left(\Omega ; \mathbb{M}_{n}\right)$, the $\mathrm{C}^{*}$-algebra of continuous functions from $\Omega$ to $\mathbb{M}_{n}$ which vanish at infinity. The norm of an element $y=\left(y_{i j}\right) \in C_{0}\left(\Omega ; \mathbb{M}_{n}\right)$ is given by

$$
\|y\|=\sup _{\omega \in \Omega}\left\|\left(y_{i j}(\omega)\right)\right\|_{\mathbb{M}_{n}} .
$$

Now let $u: E \rightarrow A$ be bounded. Then

$$
\left\|u_{n}\right\|=\sup \left\{\left\|\left(u\left(x_{i j}\right)(\omega)\right)\right\|_{\mathbb{M}_{n}}: \omega \in \Omega, x \in \mathbb{M}_{n}(E),\|x\| \leq 1\right\} .
$$

It follows that

$$
\|u\|_{c b}=\sup _{\omega \in \Omega}\left\|\delta_{\omega} \circ u\right\|_{c b},
$$

where $\delta_{\omega}: C_{0}(\Omega) \rightarrow \mathbb{C}$ is the evaluation at $\omega: \delta_{\omega}(f)=f(\omega)$. We are thus reduced to the one dimensional case.

\section{Prototypical examples of c.b. maps:}

1) Homomorphisms between $\mathbf{C}^{*}$-algebras. These maps are c.b. for they are c.p.. Moreover, they are completely contractive. Note also that an injective homomorphism is completely isometric.

2) Multiplications by bounded operators. Given $a \in B(H)$ define

$$
L_{a}: B(H) \rightarrow B(H), x \mapsto a x \quad \text { and } \quad R_{a}: B(H) \rightarrow B(H), x \mapsto x a .
$$

Then $L_{a}$ and $R_{a}$ are c.b. and

$$
\left\|L_{a}\right\|_{c b}=\left\|R_{a}\right\|_{c b}=\|a\| .
$$

Thus if $E \subset B(H)$, then $\left.L_{a}\right|_{E}$ and $\left.R_{a}\right|_{E}$ are also c.b.. 
The following theorem asserts that any c.b. map is the composition of a homomorphism, a left multiplication and a right multiplication. This is the c.b. analogue of Stinespring's factorization. We refer to [ER] and [P3] for the proof.

\section{Theorem 2.4 (Haagerup-Paulsen-Wittstock factorization)}

Let $E \subset B(H)$ and $F \subset B(K)$ be two operator spaces. Let $u: E \rightarrow F$ be a c.b. map. Then there are a Hilbert space $\widetilde{H}$, a representation $\pi: B(H) \rightarrow B(\widetilde{H})$ and two bounded operators $a, b \in B(K, \widetilde{H})$ such that

$$
u(x)=L_{b^{*}} \pi(x) R_{a}, \quad \forall x \in E .
$$

Namely, $u=\left.L_{b^{*}} \circ R_{a} \circ \pi\right|_{E}$. Moreover,

$$
\|u\|_{c b}=\inf \{\|a\|\|b\|\}
$$

where the infimum is taken over all factorizations of $u$ as above.

\section{Corollary 2.5 (Hahn-Banach type extension)}

Every c.b. map $u: E \rightarrow B(K)$ admits a c.b. extension $\tilde{u}: B(H) \rightarrow B(K)$ such that $\|\tilde{u}\|_{c b}=\|u\|_{c b}$.

Proof. Take a factorization $u=\left.L_{b^{*}} \circ R_{a} \circ \pi\right|_{E}$ such that $\|u\|_{c b}=\|a\|\|b\|$. Then $\tilde{u}=L_{b^{*}} \circ R_{a} \circ \pi$ is the desired extension of $u$.

Let $E \subset B(H)$ be an operator space. Then $E$ inherits the order of $B(H)$, which allows us to define positive and completely positive maps on $E$. Thus a map $u: E \rightarrow B(K)$ is c.p. if $u_{n}$ sends the positive part of $\mathbb{M}_{n}(E)$ into that of $\mathbb{M}_{n}(B(K))$ for every $n$.

\section{Corollary 2.6 (Decomposability of c.b. maps)}

Every c.b. map $u: E \rightarrow B(K)$ is decomposable in the sense that there are four c.p. maps $u_{k}$ such that

$$
u=u_{1}-u_{2}+i\left(u_{3}-u_{4}\right)
$$

with $\left\|u_{k}\right\|_{c b} \leq\|u\|_{c b}$.

Proof. Let $u=\left.L_{b^{*}} \circ R_{a} \circ \pi\right|_{E}$ according to Theorem 2.4. Then the corollary immediately follows from the polarization identity with

$$
u_{k}=\left.\frac{1}{4} C_{a+i^{k} b} \circ \pi\right|_{E}, \quad 1 \leq k \leq 4
$$

where $C_{a}$ is the multiplication by $a$ from the right and by $a^{*}$ from the left.

Usually, we do not distinguish completely isometric operator spaces. The distance between two operator spaces is measured by the operator space analogue of the Banach-Mazur distance in the theory of Banach spaces. The Banach-Mazur distance of two Banach spaces $E$ and $F$ is

$$
d(E, F)=\inf \left\{\left\|u^{-1}\right\|\|u\|: u: E \rightarrow F \text { is an isomorphism }\right\} .
$$

If $E$ and $F$ are isomorphic, $d(E, F)<\infty$; otherwise, $d(E, F)=\infty$.

Definition 2.7 Given two operator spaces $E$ and $F$ define

$$
d_{c b}(E, F)=\inf \left\{\left\|u^{-1}\right\|_{c b}\|u\|_{c b}: u: E \rightarrow F \text { is a complete isomorphism }\right\} .
$$

Pisier [P1] proved that if $\operatorname{dim} E=\operatorname{dim} F=n$, then $d_{c b}(E, F) \leq n$. We will see that the upper bound is attained for the pair $\left(C^{n}, R^{n}\right)$. To this end, let us introduce a general definition.

Definition 2.8 An operator space $E$ is called homogeneous if every bounded map on $E$ is c.b. and $\|u\|_{c b}=\|u\|$. $E$ is called Hilbertian if $E$ is isometric to a Hilbert space. 
It is easy to see that $\min (E)$ and $\max (E)$ are homogeneous. On the other hand, $C$ and $R$ are homogeneous Hilbertian operator spaces. We refer to Chapiter 10 of [P3] for the proofs of the following two results.

Proposition 2.9 Let $E$ and $F$ be two n-dimensional Hilbertian homogeneous operator spaces. Let $\left(e_{1}, \ldots, e_{n}\right)$ and $\left(f_{1}, \ldots, f_{n}\right)$ be orthonormal bases of $E$ and $F$, respectively. Let $u: E \rightarrow F$ be the map defined by $u\left(e_{i}\right)=f_{i}$. Then

$$
d_{c b}(E, F)=\left\|u^{-1}\right\|_{c b}\|u\|_{c b} .
$$

Corollary $2.10 d_{c b}\left(C^{n}, R^{n}\right)=n$ and $\left.d_{c b}\left(C^{n}, \min \left(\ell_{2}^{n}\right)\right)\right)=\sqrt{n}$. Consequently, $C, R$ and $\min \left(\ell_{2}\right)$ are not completely isomorphic each other.

\section{Exercices:}

1) Let $E$ be a Banach space and $F$ an operator space. Prove that any bounded maps $u: F \rightarrow$ $\min (E)$ and $v: \max (E) \rightarrow F$ are c.b. and $\|u\|_{c b}=\|u\|,\|v\|_{c b}=\|v\|$.

2) Prove that $C$ and $R$ are homogeneous.

3) Prove Corollary 2.10.

4) Let $u: C \rightarrow R$. Prove $\|u\|_{c b}=\|u\|_{H S}$, where $\|u\|_{H S}$ denotes the Hilbert-Schmidt norm of $u$ regarded as an operator on $\ell_{2}$, i.e.,

$$
\|u\|_{H S}=\left(\sum_{i=1}^{\infty}\left\|u\left(e_{i}\right)\right\|_{2}^{2}\right)^{1 / 2} .
$$

\section{Ruan's theorem: abstract operator spaces}

The definition of concrete operator spaces presented in the previous section has a major drawback: it does not allow to do basic operations on concrete operator spaces. For instance, it is not clear at all how to introduce a nice matricial structure on the Banach dual $E^{*}$ of $E$ which reflects the one of $E$. This drawback is resorbed in Ruan's definition of abstract operator spaces.

We have already seen that a concrete operator space $E \subset B(H)$ possesses a natural matricial structure inherited from that of $B(H)$ : for each $n, \mathbb{M}_{n}(E) \subset B\left(\ell_{2}^{n}(H)\right)$ is again an operator space, equipped with the norm $\|\cdot\|_{n}$ induced by that of $B\left(\ell_{2}^{n}(H)\right)$. This sequence $\left(\|\cdot\|_{n}\right)$ of matricial norms clearly satisfy the following properties

- $\left(\mathrm{R}_{1}\right):\|\alpha x \beta\|_{n} \leq\|\alpha\|\|x\|_{n}\|\beta\|, \quad \forall \alpha, \beta \in \mathbb{M}_{n}, x \in \mathbb{M}_{n}(E), n \geq 1$.

- $\left(\mathrm{R}_{2}\right):\|x \oplus y\|_{n+m} \leq \max \left(\|x\|_{n},\|y\|_{m}\right), \forall x \in \mathbb{M}_{n}(E), y \in \mathbb{M}_{m}(E), n, m \geq 1$.

Here the product is the usual matrix product. $x \oplus y$ denotes the $(n+m) \times(n+m)$-matrix

$$
\left(\begin{array}{ll}
x & 0 \\
0 & y
\end{array}\right)
$$

Theorem 3.1 (Ruan's characterization)

Let $E$ be a vector space. Assume that each $\mathbb{M}_{n}(E)$ is equipped with a norm $\|\cdot\|_{n}$. If these norms $\|\cdot\|_{n}$ satisfy Ruan's axioms $\left(R_{1}\right)$ and $\left(R_{2}\right)$, then there are a Hilbert space $H$ and a linear map $J: E \rightarrow B(H)$ such that

$$
J_{n}=\operatorname{id}_{\mathbb{M}_{n}} \otimes J: \mathbb{M}_{n}(E) \rightarrow \mathbb{M}_{n}(B(H)) \quad \text { is isometric for every } n .
$$

In other words, the sequence $\left(\|\cdot\|_{n}\right)$ comes from the operator space structure of $E$ given by the embedding $J: E \rightarrow B(H)$. 
This theorem is proved in $[\mathrm{R}]$ (see also [ER] for an alternate proof).

Definition 3.2 An (abstract) operator space is a Banach space $E$ together with a sequence $\left(\|\cdot\|_{n}\right)$ of norms satisfying $\left(\mathrm{R}_{1}\right)$ and $\left(\mathrm{R}_{2}\right)$ (with $\|\cdot\|_{1}$ equal to the original norm of $E$ ).

Henceforth, we will drop the adjective "concrete" or "abstract" by saying only operator spaces. Thus to have an operator space structure on a Banach space $E$ is to have a sequence of matricial norms verifying Ruan's axioms. In the remainder of this section we present some basic operations on operator spaces. The complex interpolation is postponed, however, to the next one, where Pisier's operator Hilbert space will be also introduced.

Spaces of c.b. maps. If $E$ and $F$ are two operator spaces, $C B(E, F)$ denotes again the space of all c.b. maps from $E$ to $F$. This is a Banach space equipped with the norm $\|\cdot\|_{c b}$. Now we wish to turn $C B(E, F)$ an operator space. Let $u=\left(u_{i j}\right) \in \mathbb{M}_{n}(C B(E, F))$. We view $u$ as a map from $E$ into $\mathbb{M}_{n}(F)$ by defining $u(x)=\left(u_{i j}(x)\right)$ for $x \in E$. Then the matricial norm on $\mathbb{M}_{n}(C B(E, F))$ is defined by

$$
\|u\|_{n}=\left\|u: E \rightarrow \mathbb{M}_{n}(F)\right\|_{c b} .
$$

Namely, we have the identification

$$
\mathbb{M}_{n}(C B(E, F))=C B\left(E, \mathbb{M}_{n}(F)\right) .
$$

It is easy to show that Ruan's axioms are verified. Thus $C B(E, F)$ becomes an operator space.

Duality. Specializing the previous discussion to $F=\mathbb{C}$, we see that $C B(E, \mathbb{C})$ is an operator space. However, Proposition 2.3 implies that $C B(E, \mathbb{C})=E^{*}$ isometrically. Therefore, $E^{*}$ becomes an operator space. The norm of $\mathbb{M}_{n}\left(E^{*}\right)$ is that of $C B\left(E, \mathbb{M}_{n}\right)$. This is usually called the standard dual of $E$. We will simply say the dual of $E$ since only standard duals are used in the sequel. The bidual $E^{* *}=\left(E^{*}\right)^{*}$ is an operator space too. Then it is easy to check that the natural inclusion $E \hookrightarrow E^{* *}$ is completely isometric. This allows to view $E$ as a subspace of $E^{* *}$.

Thus the duals of $\mathrm{C}^{*}$-algebras and the preduals of von Neumann algebras are operator spaces.

Let $u: E \rightarrow F$ be a map between two operator spaces. Then $u$ is c.b. iff its adjoint $u^{*}: F^{*} \rightarrow E^{*}$ is c.b.. If this is the case, $\|u\|_{c b}=\left\|u^{*}\right\|_{c b}$.

Quotient. Let $E$ be an operator space and $F \subset E$ a closed subspace. We equip $\mathbb{M}_{n}(E / F)$ with the quotient norm of $\mathbb{M}_{n}(E) / \mathbb{M}_{n}(F)$. Then it is easy to check that these norms satisfy $\left(\mathrm{R}_{1}\right)$ and $\left(\mathrm{R}_{2}\right)$, so $E / F$ becomes an operator space. The usual duality between subspaces and quotients in Banach space theory remains available now:

$$
F^{*}=\frac{E^{*}}{F^{\perp}} \text { and }\left(\frac{E}{F}\right)^{*}=F^{\perp} \quad \text { completely isometrically. }
$$

Direct sum. Let $\left(E_{k}\right)$ be a sequence of operator spaces, $E_{k} \subset B\left(H_{k}\right)$ (the sequence may be finite). Let $\ell_{\infty}\left(\left(E_{k}\right)\right)$ denote the space of all sequences $\left(x_{k}\right)$ with $x_{k} \in E_{k}$ such that $\sup _{k}\left\|x_{k}\right\|<\infty$. This is a Banach space when equipped with the norm

$$
\left\|\left(x_{k}\right)\right\|=\sup _{k}\left\|x_{k}\right\| .
$$

$\ell_{\infty}\left(\left(E_{k}\right)\right)$ naturally inherits a matricial structure from $\ell_{\infty}\left(\left(B\left(H_{k}\right)\right)\right)$, the latter being a $\mathrm{C}^{*}$-algebra. Thus we have

$$
\mathbb{M}_{n}\left(\ell_{\infty}\left(\left(E_{k}\right)\right)\right)=\ell_{\infty}\left(\left(\mathbb{M}_{n}\left(E_{k}\right)\right)\right) .
$$

$c_{0}\left(\left(E_{k}\right)\right)$ denotes the subspace of $\ell_{\infty}\left(\left(E_{k}\right)\right)$ consisting of all $\left(x_{k}\right)$ such that $\left\|x_{k}\right\| \rightarrow 0$.

On the other hand, we define $\ell_{1}\left(\left(E_{k}\right)\right)$ to be the space of all sequences $\left(x_{k}\right)$ with $x_{k} \in E_{k}$ such that $\sum_{k}\left\|x_{k}\right\|<\infty$. This is again a Banach space with the natural norm. Recall that

$$
\left(\ell_{1}\left(\left(E_{k}\right)\right)\right)^{*}=\ell_{\infty}\left(\left(E_{k}^{*}\right)\right) \text { isometrically. }
$$


Thus $\ell_{1}\left(\left(E_{k}\right)\right)$ is a predual of $\ell_{\infty}\left(\left(E_{k}\right)\right)$, which allows us to view $\ell_{1}\left(\left(E_{k}\right)\right)$ as an operator space too. We often use the notations

$$
\bigoplus_{k} \infty E_{k} \text { and } \bigoplus_{k} E_{k}
$$

instead of $\ell_{\infty}\left(\left(E_{k}\right)_{k}\right)$ and $\ell_{1}\left(\left(E_{k}\right)_{k}\right)$, respectively. If all $E_{k}$ are equal, these spaces are denoted by $\ell_{\infty}(E)$ and $\ell_{1}(E)$, respectively. In particular, if $E=\mathbb{C}$, we recover $\ell_{\infty}$ and $\ell_{1}$.

Let us introduce the continuous version of $c_{0}(E)$. Let $\Omega$ be a locally compact topological space and $E \subset B(H)$ an operator space. Let $C_{0}(\Omega ; E)$ denote the space of continuous functions from $\Omega$ to $E$ which vanish at infinity. $C_{0}(\Omega ; E)$ is equipped with the uniform norm

$$
\|f\|=\sup _{\omega \in \Omega}\|f(\omega)\| .
$$

Then $C_{0}(\Omega ; E) \subset C_{0}(\Omega ; B(H))$. The latter space is a $\mathrm{C}^{*}$-algebra. In this way, we turn $C_{0}(\Omega ; E)$ an operator space.

Sum and intersection. Let $\left(E_{0}, E_{1}\right)$ be a couple of operator spaces. Assume $\left(E_{0}, E_{1}\right)$ is compat$i b l e$ in the sense that both $E_{0}$ and $E_{1}$ continuously embed into a common topological vector space $V$. This allows us to define

$$
E_{0} \cap E_{1}=\left\{x \in V: x \in E_{0}, x \in E_{1}\right\}
$$

and

$$
E_{0}+E_{1}=\left\{x \in V: \exists x_{0} \in E_{0}, x_{1} \in E_{1} \text { s.t. } x=x_{0}+x_{1}\right\},
$$

equipped respectively with the intersection and sum norms

$$
\begin{gathered}
\|x\|_{E_{0} \cap E_{1}}=\max \left(\|x\|_{E_{0}},\|x\|_{E_{1}}\right), \\
\|x\|_{E_{0}+E_{1}}=\inf \left\{\left\|x_{0}\right\|_{E_{0}}+\left\|x_{1}\right\|_{E_{1}}: x=x_{0}+x_{1}, x_{0} \in E_{0}, x_{1} \in E_{1}\right\} .
\end{gathered}
$$

Note that $E_{0} \cap E_{1}$ can be regarded as the diagonal subspace of $E_{0} \oplus_{\infty} E_{1}$. On the other hand, $E_{0}+E_{1}$ is identifiable with the quotient space of $E_{0} \oplus_{1} E_{1}$ by $\Delta$, where $\Delta=\left\{\left(x_{0}, x_{1}\right): x_{0}+x_{1}=\right.$ $0\}$. Equipped with the operator space structures induced by those of $E_{0} \oplus_{\infty} E_{1}$ and $E_{0} \oplus_{1} E_{1}$, respectively, $E \cap E_{1}$ and $E_{0}+E_{1}$ become operator spaces too.

Let us consider an important example. Take the column and row spaces $C$ and $R$ and view them as compatible by identifying both of them with $\ell_{2}$ at the Banach space level. Thus if $x \in C$, we write

$$
x=\sum_{i} x_{i} e_{i 1} \quad \text { with } \quad x_{i} \in \mathbb{C} .
$$

Recall that

$$
\|x\|_{C}=\left(\sum_{i}\left|x_{i}\right|^{2}\right)^{1 / 2}=\left\|\left(x_{i}\right)\right\|_{\ell_{2}}=\left\|\sum_{i} x_{i} e_{1 i}\right\|_{R} .
$$

Then the compatibility above means that $x$ is identified with the sequence $\left(x_{i}\right)$. Accordingly, we often identify the canonical bases $\left(e_{i 1}\right)$ of $C$ and $\left(e_{1 i}\right)$ of $R$ with $\left(e_{i}\right)$ of $\ell_{2}$. Thus as Banach spaces, $C \cap R=C=R\left(=\ell_{2}\right)$. But this is no longer true in the category of operator spaces.

Let $x \in \mathbb{M}_{n}(C \cap R)=\mathbb{M}_{n}(C) \cap \mathbb{M}_{n}(R)$. Write

$$
x=\sum_{i} x_{i} \otimes e_{i} \quad \text { with } \quad x_{i} \in \mathbb{M}_{n} .
$$

Then

$$
\begin{aligned}
\|x\|_{\mathbb{M}_{n}(C \cap R)} & =\max \left(\|x\|_{\mathbb{M}_{n}(C)},\|x\|_{\mathbb{M}_{n}(R)}\right) \\
& =\max \left(\left\|\sum_{i} x_{i}^{*} x_{i}\right\|^{1 / 2},\left\|\sum_{i} x_{i} x_{i}^{*}\right\|^{1 / 2}\right) .
\end{aligned}
$$


Using this, we easily check that $C \cap R$ is not completely isomorphic to $C$. Indeed, using Proposition 2.3 , one easily shows that their $n$-dimensional versions satisfy the following

$$
d_{c b}\left(C^{n}, C^{n} \cap R^{n}\right)=\sqrt{n} .
$$

The operator space structure of $C+R$ is a little bit more complicated. Using the complete isomorphism between $E_{1} \oplus_{\infty} E_{2}$ and $E_{1} \oplus_{1} E_{2}$ (see Exercice 6 below), we deduce that for $x=$ $\sum_{i} x_{i} \otimes e_{i} \in \mathbb{M}_{n}(C+R)$

$$
\frac{1}{2}\|x\|_{\mathbb{M}_{n}(C+R)} \leq \inf \left\{\left\|\sum_{i} y_{i}^{*} y_{i}\right\|^{1 / 2}+\left\|\sum_{i} z_{i} z_{i}^{*}\right\|^{1 / 2}\right\} \leq 2\|x\|_{\mathbb{M}_{n}(C+R)},
$$

where the infimum runs over all decompositions $x_{i}=y_{i}+z_{i}$ with $y_{i}, z_{i} \in \mathbb{M}_{n}$. We will give later a complete isometric description of $C+R$ in terms of the trace class $S_{1}$.

\section{Exercices:}

1) Prove that $C^{*} \simeq R$ and $R^{*} \simeq C$ completely isometrically. More precisely, the map $\xi \in C^{*} \mapsto$ $\sum_{i} \xi\left(e_{i 1}\right) e_{1 i}$ establishes a complete isometry between $C^{*}$ and $R$.

2) Show that the natural inclusion $E \hookrightarrow E^{* *}$ is completely isometric.

3) Let $F \subset E$ be operator spaces. Prove

$$
F^{*}=\frac{E^{*}}{F^{\perp}} \quad \text { and } \quad\left(\frac{E}{F}\right)^{*}=F^{\perp} \quad \text { completely isometrically. }
$$

4) Prove that a map $u: E \rightarrow F$ is c.b. iff its adjoint $u^{*}: F^{*} \rightarrow E^{*}$ is c.b.. Moreover, $\|u\|_{c b}=$ $\left\|u^{*}\right\|_{c b}$.

5) Prove

$$
\left(c_{0}\left(\left(E_{k}\right)\right)\right)^{*}=\ell_{1}\left(\left(E_{k}^{*}\right)\right) \text { and }\left(\ell_{1}\left(\left(E_{k}\right)\right)\right)^{*}=\ell_{\infty}\left(\left(E_{k}^{*}\right)\right) \quad \text { completely isometrically. }
$$

6) Prove that the natural inclusion from $\ell_{1}\left(\left(E_{k}\right)\right)$ into $\ell_{\infty}\left(\left(E_{k}\right)\right)$ is completely contractive. Conversely, prove that the c.b. norm of the formal identity from $E_{1} \oplus_{\infty} \cdots \oplus_{\infty} E_{n}$ to $E_{1} \oplus_{1} \cdots \oplus_{1} E_{n}$ is equal to $n$.

7) Let $\left(E_{0}, E_{1}\right)$ be a compatible couple of operator spaces such that $E_{0} \cap E_{1}$ is dense in both $E_{0}$ and $E_{1}$. Prove

$$
\left(E_{0} \cap E_{1}\right)^{*}=E_{0}^{*}+E_{1}^{*}, \quad\left(E_{0}+E_{1}\right)^{*}=E_{0}^{*} \cap E_{1}^{*} \quad \text { completely isometrically. }
$$

\section{Complex interpolation and operator Hilbert spaces}

Hilbert spaces are in the center of the family of Banach spaces and play a crucial role there. It is thus natural to find their operator space analogues. One way to define operator Hilbert spaces is by complex interpolation, which is of great importance for its own right in operator space theory. Our references for this section are [P1] and [P3].

Interpolation. We first recall the definition of complex interpolation for Banach spaces. Let $\left(E_{0}, E_{1}\right)$ be a compatible couple of complex Banach spaces. Let $S=\{z \in \mathbb{C}: 0 \leq \operatorname{Re} z \leq 1\}$, a strip in $\mathbb{C}$. Let $\mathcal{F}\left(E_{0}, E_{1}\right)$ be the family of all functions $f: S \rightarrow E_{0}+E_{1}$ satisfying the following conditions:

- $f$ is continuous on $S$ and analytic in the interior of $S$; 
- $f(k+i t) \in E_{k}$ for all $t \in \mathbb{R}$ and the function $t \mapsto f(k+i t)$ is continuous from $\mathbb{R}$ to $E_{k}$ for $k=0$ and $k=1$;

- $\lim _{|t| \rightarrow \infty}\|f(k+i t)\|_{E_{k}}=0$ for $k=0$ and $k=1$.

We equip $\mathcal{F}\left(E_{0}, E_{1}\right)$ with the norm:

$$
\|f\|_{\mathcal{F}\left(E_{0}, E_{1}\right)}=\max \left\{\sup _{t \in \mathbb{R}}\|f(i t)\|_{E_{0}}, \sup _{t \in \mathbb{R}}\|f(1+i t)\|_{E_{1}}\right\} .
$$

Then it is a routine exercise to check that $\mathcal{F}\left(E_{0}, E_{1}\right)$ is a Banach space. For $0<\theta<1$ the complex interpolation space $E_{\theta}=\left(E_{0}, E_{1}\right)_{\theta}$ is defined as the space of all those $x \in E_{0}+E_{1}$ for which there exists $f \in \mathcal{F}\left(E_{0}, E_{1}\right)$ such that $f(\theta)=x$. Equipped with

$$
\|x\|_{\theta}=\inf \left\{\|f\|_{\mathcal{F}\left(E_{0}, E_{1}\right)}: f(\theta)=x, f \in \mathcal{F}\left(E_{0}, E_{1}\right)\right\},
$$

$E_{\theta}$ becomes a Banach space. Note that by the maximum principle, the map $f \mapsto f(\theta)$ is a contraction from $\mathcal{F}\left(E_{0}, E_{1}\right)$ to $E_{0}+E_{1}$. Then $\left(E_{0}, E_{1}\right)_{\theta}$ can be isometrically identified with the quotient of $\mathcal{F}\left(E_{0}, E_{1}\right)$ by the kernel of this map.

Now assume $E_{0}$ and $E_{1}$ are operator spaces. Then $\left(\mathbb{M}_{n}\left(E_{0}\right), \mathbb{M}_{n}\left(E_{1}\right)\right)$ is again compatible for any $n \geq 1$. This allows to define

$$
\mathbb{M}_{n}\left(E_{\theta}\right)=\left(\mathbb{M}_{n}\left(E_{0}\right), \mathbb{M}_{n}\left(E_{1}\right)\right)_{\theta} .
$$

It is easy to show that Ruan's axioms are satisfied, so $E_{\theta}$ is an operator space. Let us express $E_{\theta}$ as a quotient of a subspace of $C_{0}\left(\mathbb{R} ; E_{0}\right) \oplus_{\infty} C_{0}\left(\mathbb{R} ; E_{1}\right)$. Using Poisson integral, one sees that $C_{0}\left(\mathbb{R} ; E_{0}\right) \oplus_{\infty} C_{0}\left(\mathbb{R} ; E_{1}\right)$ is just the space of functions $f: S \rightarrow E_{0}+E_{1}$ satisfying the same conditions as above for $\mathcal{F}\left(E_{0}, E_{1}\right)$ but only with "analytic" replaced by "harmonic". Then $\mathcal{F}\left(E_{0}, E_{1}\right)$ is the subspace of $C_{0}\left(\mathbb{R} ; E_{0}\right) \oplus_{\infty} C_{0}\left(\mathbb{R} ; E_{1}\right)$ consisting of all analytic functions. Therefore, $E_{\theta}$ is the quotient space of $\mathcal{F}\left(E_{0}, E_{1}\right)$ by the subspace of all $f$ such that $f(\theta)=0$.

Operator Hilbert spaces. Let $H$ be a complex Hilbert space. Fixing an orthonormal basis $\left(e_{i}\right)_{i \in I}$ in $H$, we can identify $H$ with $\ell_{2}(I)$. The classical Riesz representation theorem asserts that $H^{*}$ is isometric to the conjugate $\bar{H}$ of $H$. The latter space is $H$ itself with the same norm but with conjugate multiplication: $\lambda \cdot x=\bar{\lambda} x$ for $\lambda \in \mathbb{C}$ and $x \in H$. Viewed as a vector in $\bar{H}$, a vector $x \in H$ is often denoted by $\bar{x}$. Thus if $x=\left(x_{i}\right) \in \ell_{2}(I)$, then $\bar{x}=\left(\bar{x}_{i}\right)_{i \in I}$. The map $x \mapsto \bar{x}$ establishes an anti-linear isometry between $H$ and $\bar{H}$. Consequently, $\overline{H^{*}}$ is isometric to $H$. In fact, the conjugation can be defined for any Banach space $X$. It is a well-known elementary fact that the Hilbert spaces are only Banach spaces $X$ such that $\overline{X^{*}}$ is isometric to $X$.

Now we wish to consider the operator space analogue of this property. The resulting spaces are the so-called operator Hilbert spaces, introduced by Pisier. Note that if $E$ is an operator space, so is $\bar{E}$ by defining $\mathbb{M}_{n}(\bar{E})=\overline{\mathbb{M}}_{n}(E)$.

Theorem 4.1 Let $I$ be an index set. For any $x=\sum_{i} x_{i} \otimes e_{i} \in \mathbb{M}_{n}\left(\ell_{2}(I)\right)$ define

$$
\|x\|_{n}=\left\|\sum_{i} x_{i} \otimes \bar{x}_{i}\right\|_{\mathbb{M}_{n} \otimes \overline{\mathbb{M}}_{n}}^{1 / 2}=\left\|\sum_{i} x_{i} \otimes \bar{x}_{i}\right\|_{\mathbb{M}_{n^{2}}}^{1 / 2} .
$$

Then $\left(\|\cdot\|_{n}\right)$ satisfy Ruan's axioms. The resulting operator space is denoted by $O H(I)$. Moreover, $\mathrm{OH}(I)$ is the unique, up to complete isometry, operator space structure on $\ell_{2}(I)$ such that $\overline{\mathrm{OH}(I)^{*}}$ is completely isometric to $\mathrm{OH}(I)$.

If $I=\mathbb{N}$ or $I=\{1, \ldots, n\}$, we denote $O H(I)$ simply by $O H$ of $O H^{n}$. The space $O H$ can be also obtained by interpolating the column and row spaces.

Theorem 4.2 $\mathrm{OH}=(C, R)_{\frac{1}{2}}$ completely isometrically. 


\section{Exercices:}

1) Let $\left(E_{0}, E_{1}\right)$ and $\left(F_{0}, F_{1}\right)$ be two compatible couples of operator spaces. Let $T: E_{0}+E_{1} \rightarrow$ $F_{0}+F_{1}$ be a linear map such that $\left.T\right|_{E_{k}}: E_{k} \rightarrow F_{k}$ is c.b. of cb-norm $c_{k}$ for $k=0,1$. Then $T$ is c.b. from $E_{\theta}$ to $F_{\theta}$ for any $0<\theta<1$ and of cb-norm $\leq c_{0}^{1-\theta} c_{1}^{\theta}$.

2) Show that $O H$ is homogeneous and $d_{c b}\left(C^{n}, O H^{n}\right)=\sqrt{n}$. Consequently, $O H$ is not completely isomorphic to $C$.

\section{Vector-valued noncommutative $L_{p}$-spaces}

Since operator spaces are quantized Banach spaces, noncommutative $L_{p}$-spaces are quantized $L_{p^{-}}$ spaces. Thus it is not surprising that noncommutative $L_{p}$-spaces play the role in operator space theory as the usual $L_{p}$-spaces do in the category of Banach spaces. In this section we introduce the natural operator space structures on noncommutative $L_{p}$ and Pisier's vector-valued Schatten classes. We will need essentially the cases $p=\infty$ and $p=1$ (so the case $1<p<\infty$ can be skipped). For these special cases what is presented below becomes extremely simple for noncommutative $L_{\infty}$ and $L_{1}$ are nothing but von Neumann algebras and their preduals.

5.1 Noncommutative $L_{p}$-spaces. Let $M$ be a von Neumann algebra equipped with a normal faithful tracial state $\tau$. For $1 \leq p<\infty$ and $x \in M$ define

$$
\|x\|_{p}=\left[\tau\left(|x|^{p}\right)\right]^{1 / p}, \quad \text { where } \quad|x|=\left(x^{*} x\right)^{1 / 2} .
$$

Then $\left(M,\|\cdot\|_{p}\right)$ is a normed space, whose completion is the noncommutative $L_{p}$-space associated with $(M, \tau)$, denoted by $L_{p}(M)$. By convention, $L_{\infty}(M)=M$ with the operator norm. Then for any $1 \leq p<\infty$, the dual space of $L_{p}(M)$ is $L_{p^{\prime}}(M)$ ( $p^{\prime}$ being the conjugate index of $p$ ):

$$
L_{p}(M)^{*}=L_{p^{\prime}}(M) \quad \text { isometrically }
$$

with respect to the duality bracket

$$
\langle x, y\rangle=\tau(x y), \quad x \in L_{p}(M), y \in L_{p^{\prime}}(M) .
$$

Consequently, $L_{1}(M)$ is the predual of $M$.

Let us consider two special cases. The first is where $M$ is commutative, say, $M=L_{\infty}(\Omega, \mu)$ for some probability space $(\Omega, \mu)$ ( $\mu$ can be, of course, assumed to be a $\sigma$-finite measure). Then we recover the usual $L_{p}$-spaces $L_{p}(\Omega)$. The second case concerns $M=B\left(\ell_{2}^{n}\right)$, equipped with the usual trace $\operatorname{Tr}$ on $B\left(\ell_{2}^{n}\right)$ (which can be normalized to a state if we wish). Then we get the Schatten classes $S_{p}^{n}$. The infinite dimensional algebra $B\left(\ell_{2}\right)$ is not covered by this definition since the usual trace $\operatorname{Tr}$ on $B\left(\ell_{2}\right)$ is not finite. However, it still has a nice trace in the sense that it is normal, semifinite and faithful. The preceding construction can be done for normal semifinite faithful traces too. The resulting spaces for $\left(B\left(\ell_{2}\right), \mathrm{Tr}\right)$ are the Schatten classes $S_{p}$. Recall that $S_{1}$ and $S_{2}$ are respectively the trace and Hilbert-Schmidt classes. Note that by definition $S_{\infty}$ is the whole $B\left(\ell_{2}\right)$.

The previous definition does not apply to type III von Neumann algebras. There are several equivalent constructions of noncommutative $L_{p}$-spaces in the type III case. Here we adopt the one by Kosaki $[\mathrm{Ko}]$ via complex interpolation. Let $M$ be a von Neumann algebra equipped with a normal faithful state $\varphi$. Define $L_{\infty}(M)=M$ as before and $L_{1}(M)=M_{*}$. Consider the left injection $j$ of $L_{\infty}(M)$ into $L_{1}(M)$ by $j(x)=x \varphi$. The faithfulness of $\varphi$ implies that $j$ is injective and its range is dense in $L_{1}(M)$. This injection makes $\left(L_{\infty}(M), L_{1}(M)\right)$ compatible. Thus we can consider the complex interpolation spaces between them. Now for $1<p<\infty$ define

$$
L_{p}(M)=\left(L_{\infty}(M), L_{1}(M)\right)_{\frac{1}{p}} .
$$


We refer to the survey paper [PX] for more information and historical references on noncommutative $L_{p}$-spaces.

5.2 Operator space structures on noncommutative $L_{p}$. Now we turn to describe the natural operator space structure on $L_{p}(M)$ (see [P3] for more information). For $p=\infty, L_{\infty}(M)=M$ has its natural operator space structure as a von Neumann algebra. This also yields an operator space structure on $M^{*}$, the standard dual of $M$. To deal with the case $p=1$ we consider the opposite von Neumann algebra $M^{\mathrm{op}}$ of $M . M^{\mathrm{op}}$ is the same as $M$ but with the new multiplication which is opposite to that of $M: x \cdot y$ in $M^{\text {op }}$ is equal to $y x \in M$. Note that if $M$ acts on $H$, then $M^{\text {op }}$ acts on $H^{*}$ and coincides with $\left\{x^{t}: x \in M\right\}$, where $x^{t}$ denotes the transpose (= Banach space adjoint) of $x$. It is clear that the map $x \mapsto \overline{x^{*}}$ establishes an isomorphism between $M$ and $\overline{M^{\mathrm{op}}}$. Thus $L_{1}(M)$ is isometric to $L_{1}\left(M^{\mathrm{op}}\right)$ at the Banach space level. This allows us to equip $L_{1}(M)$ with the operator space structure inherited from $\left(M^{\mathrm{op}}\right)^{*}$. The main reason for this choice is that it insures that the equality $L_{1}\left(\mathbb{M}_{n} \otimes M\right)=S_{1}^{n} \widehat{\otimes} L_{1}(M)$ (operator space projective tensor product) holds true. Finally, the operator space structure of $L_{p}(M)$ is obtained by complex interpolation. It is worth to mention that $L_{2}(M)$ is an operator Hilbert space by virtue of a theorem of Pisier.

Thus for every $\sigma$-finite measure space $(\Omega, \mu)$, the commutative $L_{p}$-spaces $L_{p}(\Omega)$ are equipped with their natural operator space structures. In particular, the $\ell_{p}$ are operator spaces and $\ell_{2}=O H$.

The same happens to the Schatten classes $S_{p}$ too. If $1 \leq p<\infty$, the dual space of $S_{p}$ is $S_{p^{\prime}}$ with respect to the so-called parallel duality bracket

$$
\langle x, y\rangle=\operatorname{Tr}\left(x y^{t}\right)=\sum_{i, j} x_{i j} y_{i j}
$$

for $x=\left(x_{i j}\right) \in S_{p}$ and $y=\left(y_{i j}\right) \in S_{p^{\prime}}$. This is due to our definition that $S_{1}$ is defined as the predual of $B\left(\ell_{2}\right)^{\text {op }}$.

5.3 Vector-valued Schatten classes. We wish to define Schatten classes with values in operator spaces. To this end we first recall the minimal tensor product in the category of operator spaces. Let $E \subset B(H)$ and $F \subset B(K)$ be two operator spaces. Let $x \in B(H)$ and $y \in B(K)$. The tensor $x \otimes y$ is an operator on the Hilbert space tensor product $H \otimes K$ defined by $x \otimes y(\xi \otimes \eta)=x(\xi) \otimes y(\eta)$. It is easy to check that $x \otimes y$ is bounded and $\|x \otimes y\|=\|x\|\|y\|$. Consequently, the algebraic tensor product $E \otimes F$ is a vector subspace of $B(H \otimes K)$. Then the minimal tensor product $E \otimes_{\min } F$ is defined to be the closure of $E \otimes F$ in $B(H \otimes K)$.

Now let $E$ be an operator space. Define $S_{\infty}[E]$ to be $S_{\infty} \otimes_{\min } E$. The elements in $S_{\infty}[E]$ are often represented as infinite matrices with entries in $E$.

To define $S_{1}[E]$ we use duality. The operator space structure to be put in $S_{1}[E]$ will be such that the dual space of $S_{1}[E]$ is $S_{\infty}\left[E^{*}\right]$. More precisely, let $u \in S_{1} \otimes E$. Write

$$
u=\sum_{k} a_{k} \otimes x_{k} \quad \text { with } \quad a_{k} \in S_{1}, x_{k} \in E .
$$

Consider $u$ as a linear functional on $S_{\infty}\left[E^{*}\right]$ as follows. For $v=\sum_{j} b_{j} \otimes \xi_{j} \in S_{\infty} \otimes E^{*}$ define

$$
\langle u, v\rangle=\sum_{k, j}\left\langle a_{k}, b_{j}\right\rangle\left\langle x_{k}, \xi_{j}\right\rangle=\sum_{k, j} \operatorname{Tr}\left(a_{k} b_{j}^{t}\right) \xi_{j}\left(x_{k}\right) .
$$

Then the norm of $u$ is defined to be the linear functional norm of $u$ on $S_{\infty}\left[E^{*}\right]$, which coincides with the norm of $u$ in $C B\left(S_{\infty}\left[E^{*}\right], \mathbb{C}\right.$ ) (see Proposition 2.3). We define $S_{1}[E]$ to be the closure of $S_{1} \otimes E$ with respect to this norm. Next, we have to introduce a norm $\|\cdot\|_{n}$ on $\mathbb{M}_{n}\left(S_{1}[E]\right)$ for any $n$. This is now easy. As before, every $u \in \mathbb{M}_{n}\left(S_{1} \otimes E\right)$ induces a linear map from $S_{\infty}\left[E^{*}\right]$ to $\mathbb{M}_{n}$. Then define

$$
\|u\|_{n}=\|u\|_{C B\left(S_{\infty}\left[E^{*}\right], \mathbb{M}_{n}\right)} .
$$


It is then routine to check that these norms satisfy Ruan's axioms. Therefore, $S_{1}[E]$ becomes an operator space.

Having defined $S_{\infty}[E]$ and $S_{1}[E]$, we define $S_{p}[E]$ by interpolation for any $1<p<\infty$ :

$$
S_{p}[E]=\left(S_{\infty}[E], S_{1}[E]\right)_{1 / p}
$$

The elements of $S_{p}[E]$ are often represented as infinite matrices with entries in $E$. It is not hard to show that finite matrices (i.e., those with only a finite number of nonzero entries) are dense in $S_{p}[E]$ for $p<\infty$.

The following theorem of Pisier is very useful. The reader is referred to [P2] for its proof.

Theorem 5.1 Let $1 \leq p<\infty$.

i) Any $x=\left(x_{i j}\right) \in S_{p}[E]$ admits a factorization $x=$ ayb with $a, b \in S_{2 p}$ and $y \in S_{\infty}[E]$. Here the product is the usual matrix product. Moreover, we have

$$
\|x\|_{S_{p}[E]}=\inf _{x=a y b}\left\{\|a\|_{2 p}\|y\|_{S_{\infty}[E]}\|b\|_{2 p}\right\} .
$$

ii) Conversely, for any $x=\left(x_{i j}\right) \in S_{\infty}[E]$

$$
\|x\|_{S_{\infty}[E]}=\sup \left\{\|a y b\|_{S_{p}[E]}: a, b \in S_{2 p},\|a\|_{2 p} \leq 1,\|b\|_{2 p} \leq 1\right\} .
$$

Corollary 5.2 Let $E$ and $F$ be two operator spaces. Let $1 \leq p<\infty$. Then a linear map $u: E \rightarrow F$ is c.b. iff

$$
\sup _{n}\left\|I_{S_{p}^{n}} \otimes u: S_{p}^{n}[E] \rightarrow S_{p}^{n}[F]\right\|<\infty
$$

moreover in this case the supremum above is equal to $\|u\|_{c b}$. Alternatively, $u$ is c.b. iff $I_{S_{p}} \otimes u$ extends to a bounded map from $S_{p}[E]$ to $S_{p}[F]$.

Proof. Assume that $u$ is c.b., i.e.,

$$
\|u\|_{c b}=\sup _{n}\left\|I_{S_{\infty}^{n}} \otimes u: S_{\infty}^{n}[E] \rightarrow S_{\infty}^{n}[F]\right\|<\infty .
$$

Let $x=\left(x_{i j}\right)$ be a matrix in $S_{p}^{n}[E]$ with norm less than 1 . Then $x$ admits a factorization $x=a y b$ with

We have

$$
\|a\|_{S_{2 p}^{n}} \leq 1, \quad\|y\|_{S_{\infty}^{n}[E]} \leq 1, \quad\|b\|_{S_{2 p}^{n}} \leq 1
$$

$$
I \otimes u(x)=a(I \otimes u)(y) b .
$$

Therefore,

$$
\|I \otimes u(x)\|_{S_{p}^{n}[F]} \leq\|a\|_{S_{2 p}^{n}}\|I \otimes u(y)\|_{S_{\infty}^{n}[F]}\|b\|_{S_{2 p}^{n}} \leq\|u\|_{c b}
$$

whence

$$
\sup _{n}\left\|I_{S_{p}^{n}} \otimes u: S_{p}^{n}[E] \rightarrow S_{p}^{n}[F]\right\| \leq\|u\|_{c b} .
$$

The converse is proved similarly.

The previous corollary is very useful notably for subspaces of noncommutative $L_{p}$-spaces for the following reason. Given $E \subset L_{p}(M)$ it is usually difficult to determine the norm of $\mathbb{M}_{n}(E)$; but it is extremely simple to describe the norm of $S_{p}[E]$, as shows the next paragraph.

We will only need the case where $E$ is a subspace of a noncommutative $L_{p}(M)$ in which the previous theory becomes much simpler. Note that there is a natural algebraic identification of $L_{p}\left(\mathbb{M}_{n} \otimes M\right)$ with $\mathbb{M}_{n}\left(L_{p}(M)\right)$. Then $S_{p}^{n}\left[L_{p}(M)\right]$ is nothing but the linear space $\mathbb{M}_{n}\left(L_{p}(M)\right)$ 
equipped with the norm of $L_{p}\left(\mathbb{M}_{n} \otimes M\right)$. More generally, if $E \subset L_{p}(M)$ is a closed subspace, the norm of $S_{p}^{n}[E]$ is induced by that of $S_{p}^{n}\left[L_{p}(M)\right]$. In the infinite dimensional case, $S_{p}\left[L_{p}(M)\right]$ is completely isometrically identified with $L_{p}\left(B\left(\ell_{2}\right) \bar{\otimes} M\right)$ for all $1 \leq p<\infty$. If $E \subset L_{p}(M)$, then $S_{p}[E]$ is the closure of $S_{p} \otimes E$ in $L_{p}\left(B\left(\ell_{2}\right) \bar{\otimes} M\right)$.

5.4 Column and row $p$-spaces. The column and row spaces, $C$ and $R$, are pillars of the whole theory of operator spaces. Recall that $C$ and $R$ are respectively the (first) column and row subspaces of $S_{\infty}$. By analogy, let $C_{p}$ (resp. $R_{p}$ ) denote the first column (resp. row) subspace of $S_{p}$. Since $S_{2}$ is an $O H$ space, $C_{2} \simeq R_{2} \simeq O H$. The $n$-dimensional versions of these spaces are denoted by $C_{p}^{n}$ and $R_{p}^{n}$.

Now let $E$ be an operator space. We denote by $C_{p}[E]$ (resp. $R_{p}[E]$ ) the closure of $C_{p} \otimes E$ (resp. $R_{p} \otimes E$ ) in $S_{p}[E]$. If $E$ is a subspace of a noncommutative $L_{p}(M)$, the norm of $C_{p}[E]$ is easy to determine. We consider only the case where $M$ is semifinite. For any finite sequence $\left(x_{k}\right) \subset E$

$$
\left\|\sum_{k} x_{k} \otimes e_{k}\right\|_{C_{p}[E]}=\left\|\left(\sum_{k} x_{k}^{*} x_{k}\right)^{1 / 2}\right\|_{L_{p}(M)},
$$

where $\left(e_{k}\right)$ denotes the canonical basis of $C_{p}$. More generally, if $a_{k} \in C_{p}$, then

$$
\left\|\sum_{k} x_{k} \otimes a_{k}\right\|_{C_{p}[E]}=\left\|\left(\sum_{k}\left\langle a_{k}, a_{j}\right\rangle x_{k}^{*} x_{j}\right)^{1 / 2}\right\|_{L_{p}(M)},
$$

where $\langle$,$\rangle denotes the scalar product in C_{p}$. (In terms of matrix product, $\left\langle a_{k}, a_{j}\right\rangle=a_{k}^{*} a_{j}$.) We also have a similar description for $R_{p}[E]$.

We end this section by describing the operator space structure of $C+R$ with help of Corollary 5.2. To this end we use the identification that $C \simeq R_{1}$ and $R \simeq C_{1}$ (see Exercice 2 below). Thus $C+R \simeq R_{1}+C_{1}$, which is the quotient of $R_{1} \oplus_{1} C_{1}$ by the subspace $\{(x, y) ; x+y=0\}$. Therefore, for any finite sequence $\left(x_{k}\right) \subset S_{1}$

$$
\begin{aligned}
\left\|\left(x_{k}\right)\right\|_{S_{1}[C+R]} & =\inf \left\{\left\|\left(y_{k}\right)\right\|_{S_{1}\left[R_{1}\right]}+\left\|\left(z_{k}\right)\right\|_{S_{1}\left[C_{1}\right]}\right\} \\
& =\inf \left\{\left\|\left(\sum_{k}\left(y_{k} y_{k}^{*}\right)^{1 / 2}\right)\right\|_{S_{1}}+\left\|\left(\sum_{k}\left(z_{k}^{*} z_{k}\right)^{1 / 2}\right)\right\|_{S_{1}}\right\},
\end{aligned}
$$

where the infimum runs over all decompositions of $x_{k}=y_{k}+z_{k}$ in $S_{1}$. It follows that $S_{1}[C+R]=$ $C_{1}\left[S_{1}\right]+R_{1}\left[S_{1}\right]$ with equal norms (even completely isometrically).

\section{Exercices:}

1) Let $\ell_{1}$ have its natural operator space structure. Let $x \in \mathbb{M}_{n}\left(\ell_{1}\right)$ with $x=\sum_{i} x_{i} \otimes e_{i}$ (with $\left(e_{i}\right)$ the canonical basis of $\ell_{1}$ ). Prove

$$
\|x\|_{\mathbb{M}_{n}\left(\ell_{1}\right)}=\sup \left\{\left\|\sum_{i} x_{i} \otimes y_{i}\right\|_{\mathbb{M}_{m n}}: y_{i} \in \mathbb{M}_{m},\left\|y_{i}\right\| \leq 1, m \in \mathbb{N}\right\} .
$$

On the other hand, show $S_{1}\left[\ell_{1}\right]=\ell_{1}\left(S_{1}\right)$ completely isometrically.

2) Let $1 \leq p \leq \infty$ and $p^{\prime}$ be the conjugate index of $p$. Prove the following completely isometric identities:

$$
\left(C_{p}\right)^{*} \cong C_{p^{\prime}} \cong R_{p} \text { and } \quad\left(R_{p}\right)^{*} \cong R_{p^{\prime}} \cong C_{p}
$$

by identifying the canonical bases in question.

3) Prove that $C_{p}$ and $R_{p}$ are homogeneous Hilbertian spaces.

4) Compute (or estimate) $d_{c b}\left(C_{p}^{n}, C_{q}^{n}\right)$ for $1 \leq p, q \leq \infty$. Then prove that $C_{p}$ and $C_{q}$ are not completely isomorphic for $p \neq q$. 


\section{Noncommutative Khintchine type inequalities}

This section is devoted to Khintchine type inequalities in the noncommutative $L_{p}$-spaces. These inequalities are of paramount importance in operator space theory and noncommutative analysis.

6.1 The classical Khintchine inequalities. Let $\left(\varepsilon_{k}\right)$ be a Rademacher sequence on a probability space $(\Omega, P)$, i.e., an independent sequence of random variables such that $P\left(\varepsilon_{k}=1\right)=P\left(\varepsilon_{k}=\right.$ $-1)=1 / 2$. The classical Khintchine inequality states that for any $1 \leq p<\infty$ and any finite sequence $\left(x_{n}\right) \subset \mathbb{C}$

$$
\left\|\sum_{k} x_{k} \varepsilon_{k}\right\|_{p} \sim_{c_{p}}\left\|\sum_{k} x_{k} \varepsilon_{k}\right\|_{2}=\left(\sum_{k}\left|x_{k}\right|^{2}\right)^{1 / 2}
$$

Here as well as in the sequel we use $A \sim_{c} B$ to abbreviate $c^{-1} B \leq A \leq c B . c_{p}$ denotes a positive constant depending only on $p$. Using the Fubini theorem, we deduce a similar inequality for coefficients $x_{n}$ in a commutative $L_{p}$-space, say, in $L_{p}(0,1)$. Namely,

$$
\left\|\sum_{k} x_{k} \varepsilon_{k}\right\|_{L_{p}\left(\Omega ; L_{p}(0,1)\right)} \sim_{c_{p}}\left\|\left(\sum_{k}\left|x_{k}\right|^{2}\right)^{1 / 2}\right\|_{L_{p}(0,1)} .
$$

Note that the norm of $L_{p}\left(\Omega ; L_{p}(0,1)\right)$ in the above equivalence can be replaced by that of $L_{q}\left(\Omega ; L_{p}(0,1)\right)$ for any $1 \leq q<\infty$. (The relevant constant then depends on $q$ too.) This is because of the so-called Khintchine-Kahane inequalities (cf. [Ka]). Let $E$ be a Banach space and $1 \leq p, q<\infty$. Then for any finite sequence $\left(x_{k}\right) \subset E$ we have

$$
\left\|\sum_{k} x_{k} \varepsilon_{k}\right\|_{L_{p}(\Omega ; E)} \sim_{c_{p, q}}\left\|\sum_{k} x_{k} \varepsilon_{k}\right\|_{L_{q}(\Omega ; E)} .
$$

Now we wish to extend (6.2) to the noncommutative setting, i.e., replacing $L_{p}(0,1)$ by a noncommutative $L_{p}$. We will consider only the case where the coefficients $x_{k}$ are in the Schatten classes $S_{p}$. All inequalities stated below are valid for general noncommutative $L_{p}$. On the other hand, we will concentrate mainly on the case $p=1$ (and the case $p=\infty$ in the free case). Thus our gaol is to find a deterministic expression for

$$
\left\|\sum x_{k} \varepsilon_{k}\right\|_{L_{p}\left(\Omega ; S_{p}\right)}
$$

In view of the square function $\left(\sum\left|x_{k}\right|^{2}\right)^{1 / 2}$ in $(6.2)$, we are naturally led to conjecture that the deterministic expression to be found should involve the term

$$
\left\|\left(\sum\left|x_{k}\right|^{2}\right)^{1 / 2}\right\|_{p}=\left\|\left(\sum x_{k}^{*} x_{k}\right)^{1 / 2}\right\|_{p} .
$$

This norm is also equal to $\left\|\left(x_{k}\right)\right\|_{C_{p}\left[S_{p}\right]}$. Here and in the sequel, we often identify a sequence $\left(x_{k}\right)$ in $E$ with the element $\sum_{k} x_{k} \otimes e_{k}$ in $C_{p}[E]$ or $R_{p}[E]$. But now because of the noncommutativity, we should also take into account the right modulus, i.e., the term

$$
\left\|\left(\sum\left|x_{k}^{*}\right|^{2}\right)^{1 / 2}\right\|_{p}=\left\|\left(\sum x_{k} x_{k}^{*}\right)^{1 / 2}\right\|_{p}=\left\|\left(x_{k}\right)\right\|_{R_{p}\left[S_{p}\right]} .
$$

Although $\|a\|_{p}=\left\|a^{*}\right\|_{p}$ for a single operator $a \in S_{p}$, the two terms above are not comparable at all if $p \neq 2$. For example, if $x_{k}=e_{k 1}$, then clearly

$$
\left\|\left(\sum_{k=1}^{n} x_{k}^{*} x_{k}\right)^{1 / 2}\right\|_{p}=n^{1 / 2} \quad \text { and } \quad\left\|\left(\sum_{k=1}^{n} x_{k} x_{k}^{*}\right)^{1 / 2}\right\|_{p}=n^{1 / p} .
$$

6.2 Column and row subspaces. In this subsection we collect some basic properties of the column and row subspaces $C_{p}\left[S_{p}\right]$ and $R_{p}\left[S_{p}\right]$. 
Proposition 6.1 i) The Hölder inequality: Let $1 \leq p, q, r \leq \infty$ such that $1 / r=1 / p+1 / q$. Then for any sequences $\left(x_{k}\right) \in C_{p}\left[S_{p}\right]$ and $\left(y_{k}\right) \in C_{q}\left[S_{q}\right]$ the series $\sum_{k} y_{k}^{*} x_{k}$ converges in $S_{r}$ (with respect to the $w^{*}$-topology if $r=\infty$ ) and

$$
\left\|\sum_{k} y_{k}^{*} x_{k}\right\|_{r} \leq\left\|\left(x_{k}\right)\right\|_{C_{p}\left[S_{p}\right]}\left\|\left(y_{k}\right)\right\|_{C_{q}\left[S_{q}\right]} .
$$

ii) Complementation: $C_{p}\left[S_{p}\right]$ and $R_{p}\left[S_{p}\right]$ are 1-complemented subspaces of $S_{p}\left(\ell_{2} \otimes \ell_{2}\right)$ for any $1 \leq p \leq \infty$. More precisely, let $P: S_{p}\left(\ell_{2} \otimes \ell_{2}\right) \rightarrow S_{p}\left(\ell_{2} \otimes \ell_{2}\right)$ be defined by $P(x)=x e$, where $e=1 \otimes e_{11}$. Then $P$ is a contractive projection from $S_{p}\left(\ell_{2} \otimes \ell_{2}\right)$ onto $C_{p}\left[S_{p}\right]$.

iii) Duality: Let $1 \leq p<\infty$, and let $p^{\prime}$ be the conjugate index of $p$. Then

$$
C_{p}\left[S_{p}\right]^{*}=C_{p^{\prime}}\left[S_{p^{\prime}}\right] \text { and } R_{p}\left[S_{p}\right]^{*}=R_{p^{\prime}}\left[S_{p^{\prime}}\right]
$$

isometrically with respect to the anti-linear duality bracket:

$$
\left\langle\left(x_{k}\right),\left(y_{k}\right)\right\rangle \mapsto \sum_{k \geq 1} \operatorname{Tr}\left(y_{k}^{*} x_{k}\right) .
$$

Proof. i) Given $\left(x_{k}\right) \subset S_{p}$ define

$$
T\left(\left(x_{k}\right)\right)=\left(\begin{array}{ccc}
x_{1} & 0 & \cdots \\
x_{2} & 0 & \cdots \\
\vdots & \vdots &
\end{array}\right) .
$$

We view $T\left(\left(x_{k}\right)\right)$ as an element in $S_{p}\left[S_{p}\right]=S_{p}\left(\ell_{2} \otimes \ell_{2}\right)$. Now let $\left(y_{k}\right) \subset S_{q}$ be another finite sequence. Then

$$
\sum_{k} y_{k}^{*} x_{k}=T\left(\left(y_{k}\right)\right)^{*} T\left(\left(x_{k}\right)\right) .
$$

So the desired inequality follows from the Hölder inequality in $S_{p}\left(\ell_{2} \otimes \ell_{2}\right)$.

ii) This is obvious. Note that $P(x)$ is the matrix whose first column is that of $x$ and all others are zero.

iii) Using i) one sees that the duality is well-defined. The two duality equalities are immediate consequences of ii) and the duality between $S_{p}\left(\ell_{2} \otimes \ell_{2}\right)$ and $S_{p^{\prime}}\left(\ell_{2} \otimes \ell_{2}\right)$.

Proposition 6.2 Let $1 \leq p \leq \infty$ and $\left(x_{k}\right) \subset S_{p}$ be a finite sequence.

i) If $2 \leq p \leq \infty$, then

$$
\max \left\{\left\|\left(x_{k}\right)\right\|_{C_{p}\left[S_{p}\right]},\left\|\left(x_{k}\right)\right\|_{R_{p}\left[S_{p}\right]}\right\} \leq\left(\sum_{k}\left\|x_{k}\right\|_{p}^{2}\right)^{1 / 2} .
$$

ii) If $1 \leq p \leq 2$, then

$$
\min \left\{\left\|\left(x_{k}\right)\right\|_{C_{p}\left[S_{p}\right]},\left\|\left(x_{k}\right)\right\|_{R_{p}\left[S_{p}\right]}\right\} \geq\left(\sum_{k}\left\|x_{k}\right\|_{p}^{2}\right)^{1 / 2} .
$$

Proof. i) Let $p \geq 2$. By the triangle inequality in $S_{p / 2}$ we have

$$
\left\|\left(x_{k}\right)\right\|_{C_{p}\left[S_{p}\right]}^{2}=\left\|\sum_{k}\left|x_{k}\right|^{2}\right\|_{p / 2} \leq \sum_{k}\left\|x_{k}\right\|_{p}^{2} .
$$

Passing to adjoints we get the inequality on the row norm.

ii) This follows from i) by duality. 
Corollary 6.3 i) Let $(\Sigma, \mu)$ be a measure space, and let $f$ belong to the algebraic tensor product $L_{2}(\Sigma) \otimes S_{p}$. Then if $2 \leq p \leq \infty$,

$$
\max \left\{\left\|\left[\int_{\Sigma} f(t)^{*} f(t) d \mu(t)\right]^{1 / 2}\right\|_{p},\left\|\left[\int_{\Sigma} f(t) f(t)^{*} d \mu(t)\right]^{1 / 2}\right\|_{p}\right\} \leq\|f\|_{L_{2}\left(\Sigma ; S_{p}\right)} .
$$

and if $1 \leq p \leq 2$,

$$
\|f\|_{L_{2}\left(\Sigma ; S_{p}\right)} \leq \min \left\{\left\|\left[\int_{\Sigma} f(t)^{*} f(t) d \mu(t)\right]^{1 / 2}\right\|_{p},\left\|\left[\int_{\Sigma} f(t) f(t)^{*} d \mu(t)\right]^{1 / 2}\right\|_{p}\right\} .
$$

ii) In particular, if $\left(\varphi_{k}\right)$ is an orthonormal sequence in $L_{2}(\Sigma)$ and $\left(x_{k}\right)$ a finite sequence in $S_{p}$, then for $2 \leq p \leq \infty$

$$
\max \left\{\left\|\left(x_{k}\right)\right\|_{C_{p}\left[S_{p}\right]},\left\|\left(x_{k}\right)\right\|_{R_{p}\left[S_{p}\right]}\right\} \leq\left\|\sum_{k} x_{k} \varphi_{k}\right\|_{L_{2}\left(\Sigma ; S_{p}\right)} ;
$$

and for $1 \leq p \leq 2$

$$
\left\|\sum_{k} x_{k} \varphi_{k}\right\|_{L_{2}\left(\Sigma ; S_{p}\right)} \leq \inf \left\{\left\|\left(y_{k}\right)\right\|_{C_{p}\left[S_{p}\right]}+\left\|\left(z_{k}\right)\right\|_{R_{p}\left[S_{p}\right]}\right\},
$$

where the infimum runs over all decompositions $x_{k}=y_{k}+z_{k}$ with $y_{k}$ and $z_{k}$ in $S_{p}$.

Proof. i) Note that since $f \in L_{2}(\Sigma) \otimes S_{p}$, the two integrals

$$
\int_{\Sigma} f(t)^{*} f(t) d \mu(t) \quad \text { and } \quad \int_{\Sigma} f(t) f(t)^{*} d \mu(t)
$$

are well-defined and belong to $S_{p / 2}$. The desired inequalities follow from the corresponding ones in the previous proposition.

ii) The first inequality immediately follows from i) above. To prove the second take a decomposition $x_{k}=y_{k}+z_{k}$. Then again by the previous proposition

$$
\begin{aligned}
\left\|\sum_{k} x_{k} \varphi_{k}\right\|_{L_{2}\left(\Sigma ; S_{p}\right)} & \leq\left\|\sum_{k} y_{k} \varphi_{k}\right\|_{L_{2}\left(\Sigma ; S_{p}\right)}+\left\|\sum_{k} z_{k} \varphi_{k}\right\|_{L_{2}\left(\Sigma ; S_{p}\right)} \\
& \leq\left\|\left(y_{k}\right)\right\|_{C_{p}\left[S_{p}\right]}+\left\|\left(z_{k}\right)\right\|_{R_{p}\left[S_{p}\right]} ;
\end{aligned}
$$

whence the desired inequality.

Recall that $(C, R)$ is considered as a compatible pair by identifying their canonical bases with that of $\ell_{2}$. This identification is extended to all spaces $C_{p}$ and $R_{p}$. Thus any pair $\left(C_{p}, R_{q}\right)$ is also compatible. Then the maximum and infimum in Corollary 6.3, ii) are respectively $\left\|\left(x_{k}\right)\right\|_{C_{p}\left[S_{p}\right] \cap R_{p}\left[S_{p}\right]}$ and $\left\|\left(x_{k}\right)\right\|_{C_{p}\left[S_{p}\right]+R_{p}\left[S_{p}\right]}$. For notational simplicity, we introduce the following

Definition 6.4 For $1 \leq p \leq \infty$ define

$$
C R_{p}\left[S_{p}\right]=C_{p}\left[S_{p}\right] \cap R_{p}\left[S_{p}\right] \text { if } p \geq 2 \text { and } C R_{p}\left[S_{p}\right]=C_{p}\left[S_{p}\right]+R_{p}\left[S_{p}\right] \text { if } p<2 .
$$

Since $C_{p}\left[S_{p}\right]=S_{p}\left[C_{p}\right]$ and $R_{p}\left[S_{p}\right]=S_{p}\left[R_{p}\right]$,

$$
C_{p}\left[S_{p}\right] \cap R_{p}\left[S_{p}\right]=S_{p}\left[C_{p} \cap R_{p}\right] \quad \text { and } \quad C_{p}\left[S_{p}\right]+R_{p}\left[S_{p}\right]=S_{p}\left[C_{p}+R_{p}\right]
$$

with equivalent norms; moreover, the equivalence constants are controlled by a universal one. By Corollary 5.2, these identities are also completely isomorphic. Thus if we define $C R_{p}=C_{p} \cap R_{p}$ for $p \geq 2$ and $C R_{p}=C_{p}+R_{p}$ for $p<2$, we obtain $C R_{p}\left[S_{p}\right]=S_{p}\left[C R_{p}\right]$ with complete equivalent norms.

We are now well prepared for our noncommutative Khintchine inequalities.

6.3 Rademacher sequences. The following is the noncommutative Khintchine inequality for Rademacher variables $\left(\varepsilon_{k}\right)$ due to Lust-Piquard and Pisier. 
Theorem 6.5 Let $1 \leq p<\infty$ and $\left(x_{k}\right)$ be a finite sequence in $S_{p}$. Then

$$
\left\|\sum_{k} x_{k} \varepsilon_{k}\right\|_{L_{p}\left(\Omega ; S_{p}\right)} \sim_{c_{p}}\left\|\left(x_{k}\right)\right\|_{C R_{p}\left[S_{p}\right]} .
$$

More precisely,

i) if $2 \leq p<\infty$, then

$$
\left\|\left(x_{k}\right)\right\|_{C R_{p}\left[S_{p}\right]} \leq\left\|\sum_{k} x_{k} \varepsilon_{n}\right\|_{L_{p}\left(\Omega ; S_{p}\right)} \leq c \sqrt{p}\left\|\left(x_{k}\right)\right\|_{C R_{p}\left[S_{p}\right]}
$$

ii) if $1 \leq p<2$, then

$$
c^{-1}\left\|\left(x_{k}\right)\right\|_{C R_{p}\left[S_{p}\right]} \leq\left\|\sum_{k} x_{k} \varepsilon_{k}\right\|_{L_{p}\left(\Omega ; S_{p}\right)} \leq\left\|\left(x_{k}\right)\right\|_{C R_{p}\left[S_{p}\right]},
$$

where $c$ is a universal positive constant.

Proof. The lower estimate in i) and the upper estimate in ii) follow from Corollary 6.3. We will prove only the lower estimate in ii) for $p=1$, following the recent approach of Haagerup and Musat $[\mathrm{HM}]$. The reader is referred to $[\mathrm{LPP}]$ for the proof of all remaining cases and to [P2] for the optimal order of the best constant for the upper estimate in i).

By duality, the lower estimate of ii) for $p=1$ is equivalent to the following statement:

(*) For any finite sequence $\left(x_{k}\right) \subset S_{\infty}$ there exists a function $f \in L_{\infty}\left(\Omega ; S_{\infty}\right)$ such that

$$
\widehat{f}\left(\varepsilon_{k}\right)=x_{k} \quad \text { and } \quad\|f\|_{L_{\infty}\left(\Omega ; S_{\infty}\right)} \leq c\left\|\left(x_{k}\right)\right\|_{C R_{\infty}\left[S_{\infty}\right]},
$$

where $\widehat{f}\left(\varepsilon_{k}\right)=\mathbb{E}\left(f \varepsilon_{k}\right)$ with $\mathbb{E}$ denoting the expectation on the probability space $(\Omega, P)$.

Let $x_{k} \in S_{\infty}$ be selfadjoint and such that $\left\|\left(x_{k}\right)\right\|_{C R_{\infty}\left[S_{\infty}\right]} \leq 1$. Let

$$
g=\sum_{k} \varepsilon_{k} x_{k} .
$$

Then

$$
g^{2}=\sum_{k} x_{k}^{2}+\sum_{j<k} \varepsilon_{j} \varepsilon_{k}\left(x_{j} x_{k}+x_{k} x_{j}\right)
$$

and

$$
\begin{aligned}
\mathbb{E}\left(g^{4}\right) & =\left(\sum_{k} x_{k}^{2}\right)^{2}+\sum_{j<k}\left(x_{j} x_{k}+x_{k} x_{j}\right)^{2} \\
& \leq 1+2 \sum_{j<k}\left(x_{j} x_{k}^{2} x_{j}+x_{k} x_{j}^{2} x_{k}\right) \\
& =1+2 \sum_{j} x_{j}\left(\sum_{k \neq j} x_{k}^{2}\right) x_{j} \leq 3 .
\end{aligned}
$$

Now let $\lambda$ be a positive number to be determined later. Set

$$
f_{\lambda}=g \mathbb{1}_{[-\lambda, \lambda]}(g) \text { and } g_{\lambda}=g-f_{\lambda},
$$

where $\mathbb{1}_{[-\lambda, \lambda]}(g)$ denotes the spectral projection of $g$ corresponding to the interval $[-\lambda, \lambda]$. By definition, $\left\|f_{\lambda}\right\|_{L_{\infty}\left(\Omega ; S_{\infty}\right)} \leq \lambda$. On the other hand, letting $z_{k}=\mathbb{E}\left(g_{\lambda} \varepsilon_{k}\right)$, we have

$$
\sum_{k} z_{k}^{2} \leq \mathbb{E}\left(g_{\lambda}^{2}\right) .
$$


However,

$$
\lambda^{2} g_{\lambda}^{2} \leq g^{4} .
$$

Therefore, combining the preceding inequalities, we find

$$
\left\|\left(\sum_{k} z_{k}^{2}\right)^{1 / 2}\right\|_{\infty} \leq\left\|\left(\mathbb{E}\left(g_{\lambda}^{2}\right)\right)^{1 / 2}\right\|_{L_{\infty}\left(\Omega ; S_{\infty}\right)} \leq \lambda^{-1}\left\|\left(\mathbb{E}\left(g^{4}\right)\right)^{1 / 2}\right\|_{L_{\infty}\left(\Omega ; S_{\infty}\right)} \leq \frac{\sqrt{3}}{\lambda} .
$$

For $\lambda=2 \sqrt{3}$ let

$$
f^{(0)}=f_{\lambda}, \quad x_{k}^{(0)}=\mathbb{E}\left(f^{(0)} \varepsilon_{k}\right), \quad z_{k}^{(0)}=z_{k} .
$$

Then

$$
x_{k}=x_{k}^{(0)}+z_{k}^{(0)}, \quad\left\|f^{(0)}\right\|_{L_{\infty}\left(\Omega ; S_{\infty}\right)} \leq 2 \sqrt{3}, \quad\left\|\left(z_{k}^{(0)}\right)\right\|_{C R_{\infty}\left[S_{\infty}\right]} \leq \frac{1}{2} .
$$

Repeating the same argument with $2 z_{k}^{(0)}$ instead of $x_{k}$, we find a function $f^{(1)}$ and a finite sequence $\left(z_{k}^{(1)}\right)$ such that

$$
2 z_{k}^{(0)}=x_{k}^{(1)}+z_{k}^{(1)}, \quad\left\|f^{(1)}\right\|_{L_{\infty}\left(\Omega ; S_{\infty}\right)} \leq 2 \sqrt{3}, \quad\left\|\left(z_{k}^{(1)}\right)\right\|_{C R_{\infty}\left[S_{\infty}\right]} \leq \frac{1}{2},
$$

where

$$
x_{k}^{(1)}=\mathbb{E}\left(f^{(1)} \varepsilon_{k}\right) .
$$

Continuing this procedure we obtain a sequence $\left(f^{(n)}\right)$ of functions and a sequence $\left(x_{k}^{(n)}\right)$ in $S_{\infty}$ such that

$$
x_{k}=\sum_{n \geq 0} 2^{-n} x_{k}^{(n)}, \quad\left\|f^{(n)}\right\|_{L_{\infty}\left(\Omega ; S_{\infty}\right)} \leq 2 \sqrt{3}, \quad x_{k}^{(n)}=\mathbb{E}\left(f^{(n)} \varepsilon_{k}\right) .
$$

Put

$$
f=\sum_{n \geq 0} 2^{-n} f^{(n)}
$$

Then

$$
\|f\|_{L_{\infty}\left(\Omega ; S_{\infty}\right)} \leq 4 \sqrt{3} \text { and } x_{k}=\mathbb{E}\left(f \varepsilon_{k}\right) .
$$

Thus the statement $(*)$ is proved for selfadjoint $x_{k}$. The general case is easily reduced to the selfadjoint case by decomposing each $x_{k}$ into its real and imaginary parts. Note that the final constant $c$ obtained in this way is $8 \sqrt{3}$. We refer to [HM] for a more careful argument which yields a constant $\sqrt{3}$.

Remark 6.6 Let $\mathcal{R}_{p}$ be the closed subspace of $L_{p}(\Omega)$ generated by the $\varepsilon_{k}$. Using Corollary 5.2, we can rephrase Theorem 6.5 as the fact that $\mathcal{R}_{p}$ is completely isomorphic to $C R_{p}$. This remark also applies to Theorems 6.8 and 6.9 below.

Remark 6.7 The Rademacher sequence $\left(\varepsilon_{k}\right)$ can be replaced by a standard Gaussian sequence. More generally, let $\left(\varphi_{k}\right)$ be an independent sequence of random variables on $(\Omega, P)$. Assume that $\left(\varphi_{k}\right)$ is symmetric in the sense that $\left(\varphi_{k}\right)$ has the same distribution as $\left( \pm \varphi_{k}\right)$ for any sequence of signs. Assume further that

$$
0<\inf _{k}\left\|\varphi_{k}\right\|_{p}, \quad \sup _{k}\left\|\varphi_{k}\right\|_{2}<\infty \quad \text { for } 1 \leq p<2
$$

and

$$
0<\inf _{k}\left\|\varphi_{k}\right\|_{2}, \quad \sup _{k}\left\|\varphi_{k}\right\|_{p}<\infty \text { for } 2 \leq p<\infty .
$$

Then Theorem 6.5 holds for $\left(\varphi_{k}\right)$ instead of $\left(\varepsilon_{k}\right)$ with relevant constants depending on $\left(\varphi_{k}\right)$. 
Since now we are in the noncommutative setting, it is natural to consider Khintchine type inequalities for noncommutative random variables instead of $\left(\varepsilon_{k}\right)$. We first consider the tracial case by giving two important examples. Namely, free generators and semicircular systems. We start with free generators.

6.4 Free generators. Consider a discrete group $G$. Let $\left(\delta_{g}\right)_{g \in G}$ be the canonical basis of $\ell_{2}(G)$, i.e., $\delta_{g}$ is the function on $G$ that takes value 1 at $g$ and zero elsewhere. Let $\lambda: G \rightarrow B\left(\ell_{2}(G)\right)$ be the left regular representation. Namely, for any $g \in G, \lambda(g)$ is the unitary operator on $\ell_{2}(G)$ defined by

$$
(\lambda(g) \varphi)(h)=\varphi\left(g^{-1} h\right), \quad h, g \in G, \varphi \in \ell_{2}(G) .
$$

Note that $\lambda(g) \delta_{h}=\delta_{g h}$ for all $g, h \in G$. Then the group von Neumann algebra $V N(G)$ is the von Neumann subalgebra of $B\left(\ell_{2}(G)\right)$ generated by $\{\lambda(g): g \in G\}$. Namely, $V N(G)$ is the w*-closure of all finite sums $\sum \alpha_{g} \lambda(g)$ with $\alpha_{g} \in \mathbb{C}$. $V N(G)$ also coincides with the left convolution algebra of $\ell_{2}(G)$. Recall that if $\varphi, \psi \in \ell_{2}(G)$, their convolution is defined by

$$
\varphi * \psi(g)=\sum_{h \in G} \varphi(h) \psi\left(h^{-1} g\right), \quad g \in G .
$$

Then $x \in V N(G)$ iff there exists $\varphi \in \ell_{2}(G)$ such that $x \psi=\varphi * \psi$ for every $\psi \in \ell_{2}(G)$. Let $\tau_{G}$ be the vector state on $V N(G)$ determined by $\delta_{e}$, i.e., $\tau_{G}(x)=\left\langle x \delta_{e}, \delta_{e}\right\rangle$ for any $x \in V N(G)$. Then it is easy to check that $\tau_{G}$ is faithful and tracial.

If we identify an operator $x \in V N(G)$ with its symbol $x \delta_{e}$ in $\ell_{2}(G)$, then $L_{2}(V N(G))$ is nothing but $\ell_{2}(G) . L_{1}(V N(G))$ is traditionally called the Fourier algebra of $G$ and denoted by $A(G)$. Since an operator in $L_{1}(V N(G))$ is a product of two operators in $L_{2}(V N(G)$ ), a function $\varphi$ on $G$ belongs $A(G)$ iff there exist two functions $\psi, \rho \in \ell_{2}(G)$ such that $\varphi=\psi \rho$. We refer to [KR] for more information.

If $G$ is abelian, $V N(G)$ ie equal to $L_{\infty}(\widehat{G})$, so is commutative, where $\widehat{G}$ is the dual group of $G$.

An important example of non abelian groups is a free group $\mathbb{F}$ on $n$ generators $\left(g_{k}\right)$ with $n \in \mathbb{N} \cup\{\infty\}$. The sequence $\left(\lambda\left(g_{k}\right)\right)_{k}$ of the unitary operators given by the generators is of particular interest. With a slight abuse of terminology, we will also call it a sequence of free generators. Note that $\left(\lambda\left(g_{k}\right)\right)_{k}$ is orthonormal in $L_{2}(V N(\mathbb{F}))$. Let us determine its linear span in $V N(\mathbb{F})$. To this end let $F_{k}$ be the closed subspace of $\ell_{2}(\mathbb{F})$ generated by all those basic vectors $\delta_{g}$ for which $g$ is a reduced word starting with $g_{k}^{-1}$. The $F_{k}, k=1,2, \ldots$, are mutually orthogonal. Let $\mathcal{F}_{k}$ be the orthogonal projection from $\ell_{2}(\mathbb{F})$ onto $F_{k}$. Now, given a finite sequence $\left(\alpha_{k}\right) \subset \mathbb{C}$ write

$$
\sum_{k} \alpha_{k} \lambda\left(g_{k}\right)=\sum_{k} \alpha_{k} \lambda\left(g_{k}\right) \mathcal{F}_{k}+\sum_{k} \alpha_{k} \lambda\left(g_{k}\right) \mathcal{F}_{k}^{\perp}
$$

By the mutual orthogonality of the $\mathcal{F}_{k}$, we have

$$
\begin{aligned}
\left\|\sum_{k} \alpha_{k} \lambda\left(g_{k}\right) \mathcal{F}_{k}\right\|_{\infty}^{2} & =\left\|\left(\sum_{k} \alpha_{k} \lambda\left(g_{k}\right) \mathcal{F}_{k}\right)\left(\sum_{k} \alpha_{k} \lambda\left(g_{k}\right) \mathcal{F}_{k}\right)^{*}\right\|_{\infty} \\
& =\left\|\sum_{k}\left|\alpha_{k}\right|^{2} \lambda\left(g_{k}\right) \mathcal{F}_{k} \lambda\left(g_{k}\right)^{*}\right\|_{\infty} \leq \sum_{k}\left|\alpha_{k}\right|^{2} .
\end{aligned}
$$

To treat another term, observe that for $k \neq j$ the range of $\lambda\left(g_{k}\right)^{*} \lambda\left(g_{j}\right) \mathcal{F}_{j}^{\perp}$ is contained in $F_{k}$, so $\mathcal{F}_{k}^{\perp} \lambda\left(g_{k}\right)^{*} \lambda\left(g_{j}\right) \mathcal{F}_{j}^{\perp}=0$. Then it follows that

$$
\begin{aligned}
\left\|\sum_{k} \alpha_{k} \lambda\left(g_{k}\right) \mathcal{F}_{k}^{\perp}\right\|_{\infty}^{2} & =\left\|\left(\sum_{k} \alpha_{k} \lambda\left(g_{k}\right) \mathcal{F}_{k}^{\perp}\right)^{*}\left(\sum_{k} \alpha_{k} \lambda\left(g_{k}\right) \mathcal{F}_{k}^{\perp}\right)\right\|_{\infty} \\
& =\left\|\sum_{k}\left|\alpha_{k}\right|^{2} \mathcal{F}_{k}^{\perp}\right\|_{\infty} \leq \sum_{k}\left|\alpha_{k}\right|^{2} .
\end{aligned}
$$


Therefore,

$$
\left\|\sum_{k} \alpha_{k} \lambda\left(g_{k}\right)\right\|_{\infty} \leq 2\left(\sum_{k}\left|\alpha_{k}\right|^{2}\right)^{1 / 2} .
$$

The converse inequality with constant 1 is obvious for

$$
\left\|\sum_{k} \alpha_{k} \lambda\left(g_{k}\right)\right\|_{\infty} \geq\left\|\sum_{k} \alpha_{k} \lambda\left(g_{k}\right)\right\|_{2}=\left(\sum_{k}\left|\alpha_{k}\right|^{2}\right)^{1 / 2} .
$$

Consequently, for any $1 \leq p \leq \infty$ the closed subspace generated by the $\lambda\left(g_{k}\right)$ in $L_{p}(V N(\mathbb{F}))$ is isomorphic to $\ell_{2}$. More precisely, for any finite sequence $\left(\alpha_{k}\right) \subset \mathbb{C}$,

$$
\left\|\sum_{k} \alpha_{k} \lambda\left(g_{k}\right)\right\|_{p} \sim_{c}\left(\sum_{k}\left|\alpha_{k}\right|^{2}\right)^{1 / 2},
$$

where $c$ is a universal constant. This inequality remains true if the scalar coefficients $\left(\alpha_{k}\right)$ are replaced by operator coefficients. The resulting inequalities are the Khintchine inequality for free generators. In the following statement $L_{p}\left(B\left(\ell_{2}\right) \bar{\otimes} V N(\mathbb{F})\right)$ is the noncommutative $L_{p}$-space associated with the von Neumann tensor product $B\left(\ell_{2}\right) \bar{\otimes} V N(\mathbb{F})$, equipped with the tensor trace $\operatorname{Tr} \otimes \tau_{\mathbb{F}}$, which is a normal semifinite faithful trace. Note that

$$
L_{p}\left(B\left(\ell_{2}\right) \bar{\otimes} V N(\mathbb{F})\right)=S_{p}\left[L_{p}(V N(\mathbb{F}))\right] .
$$

The following theorem is due to Haagerup/Pisier [HP] for $p=\infty, 1$ and to Pisier [P2] for $1<p<\infty$.

Theorem 6.8 Let $1 \leq p \leq \infty$ and $\left(x_{k}\right)$ be a finite sequence in $S_{p}$. Then

$$
\left\|\sum_{k} x_{k} \otimes \lambda\left(g_{k}\right)\right\|_{L_{p}\left(B\left(\ell_{2}\right) \bar{\otimes} V N(\mathbb{F})\right)} \sim_{c}\left\|\left(x_{k}\right)\right\|_{C R_{p}\left[S_{p}\right]}
$$

with a universal constant c. Moreover, the closed subspace of $L_{p}(V N(\mathbb{F}))$ generated by the $\lambda\left(g_{k}\right)$ is completely complemented in $L_{p}(V N(\mathbb{F}))$ with relevant constant $\leq 2$.

Proof. Here we prove (6.3) only in the cases $p=\infty, 1$ and the complementation assertion. The remaining cases are postponed to subsection 6.6. For $p=\infty$ we have the following inequalities

$$
\left\|\left(x_{k}\right)\right\|_{C R_{\infty}\left[S_{\infty}\right]} \leq\left\|\sum_{k} x_{k} \otimes \lambda\left(g_{k}\right)\right\|_{B\left(\ell_{2}\right) \bar{\otimes} V N(\mathbb{F})} \leq 2\left\|\left(x_{k}\right)\right\|_{C R_{\infty}\left[S_{\infty}\right]}
$$

for any finite sequence $\left(x_{k}\right) \subset S_{\infty}$. The proof of the second inequality above is the same as in the scalar case. Let us show the first one. Take a unit vector $\xi \in \ell_{2}$. Then

$$
\begin{aligned}
\left\|\sum_{k} x_{k} \otimes \lambda\left(g_{k}\right)\right\|_{\infty}^{2} & \geq\left\langle\sum_{k} x_{k} \otimes \lambda\left(g_{k}\right)\left(\xi \otimes \delta_{e}\right), \sum_{k} x_{k} \otimes \lambda\left(g_{k}\right)\left(\xi \otimes \delta_{e}\right)\right\rangle \\
& =\left\langle\sum_{k} x_{k}(\xi) \otimes \delta_{g_{k}}, \sum_{k} x_{k}(\xi) \otimes \delta_{g_{k}}\right\rangle \\
& =\sum_{k}\left\langle x_{k}(\xi), x_{k}(\xi)\right\rangle=\left\langle\sum_{k} x_{k}^{*} x_{k}(\xi), \xi\right\rangle ;
\end{aligned}
$$

whence

$$
\left\|\left(\sum_{k} x_{k}^{*} x_{k}\right)^{\frac{1}{2}}\right\|_{\infty} \leq\left\|\sum_{k} x_{k} \otimes \lambda\left(g_{k}\right)\right\|_{\infty}
$$

Taking adjoints, we find

$$
\left\|\left(\sum_{k} x_{k} x_{k}^{*}\right)^{\frac{1}{2}}\right\|_{\infty} \leq\left\|\sum_{k} x_{k} \otimes \lambda\left(g_{k}\right)\right\|_{\infty}
$$


Therefore, the lower estimate of (6.4) is proved.

Dualizing (6.4), we get the case $p=1$ :

$$
\frac{1}{2}\left\|\left(x_{k}\right)\right\|_{C R_{1}\left[S_{1}\right]} \leq\left\|\sum_{k} x_{k} \otimes \lambda\left(g_{k}\right)\right\|_{L_{1}\left(B\left(\ell_{2}\right) \bar{\otimes} V N(\mathbb{F})\right)} \leq\left\|\left(x_{k}\right)\right\|_{C R_{1}\left[S_{1}\right]}
$$

for any finite sequence $\left(x_{k}\right) \subset S_{1}$. Indeed, let $\left(y_{k}\right) \subset S_{\infty}$ be such that

$$
\left\|\left(x_{k}\right)\right\|_{C R_{1}\left[S_{1}\right]}=\sum_{k} \operatorname{Tr}\left(y_{k}^{*} x_{k}\right) \quad \text { and } \quad\left\|\left(y_{k}\right)\right\|_{C R_{\infty}\left[S_{\infty}\right]} \leq 1
$$

(see Proposition 6.1 iii)). Set

$$
x=\sum_{k} x_{k} \otimes \lambda\left(g_{k}\right) \in L_{1}\left(B\left(\ell_{2}\right) \bar{\otimes} V N(\mathbb{F})\right) \quad \text { and } \quad y=\sum_{k} y_{k} \otimes \lambda\left(g_{k}\right) \in B\left(\ell_{2}\right) \bar{\otimes} V N(\mathbb{F}) .
$$

Then by (6.4)

$$
\sum_{k} \operatorname{Tr}\left(y_{k}^{*} x_{k}\right)=\operatorname{Tr} \otimes \tau_{\mathbb{F}}\left(y^{*} x\right) \leq\|y\|_{\infty}\|x\|_{1} \leq 2\left\|\left(y_{k}\right)\right\|_{C R_{\infty}\left[S_{\infty}\right]}\|x\|_{1} \leq 2\|x\|_{1} .
$$

This is the lower estimate of (6.5). To show the upper estimate we consider the projection $P$ from the algebra of all finite sums $\sum \alpha_{g} \lambda(g)$ with $\alpha_{g} \in \mathbb{C}$ onto the linear span of the $\lambda\left(g_{k}\right)$. Let $Q=I-P$. It is easy to see that $P(x)$ and $Q(x)$ are orthogonal relative to the scalar product of $L_{2}(V N(\mathbb{F}))$. Thus the extension of $P$ on $L_{2}(V N(\mathbb{F}))$ is the orthogonal projection from $L_{2}(V N(\mathbb{F}))$ onto the closed subspace generated by the $\lambda\left(g_{k}\right)$. Now let $x=\sum_{g \in \mathbb{F}} x_{g} \otimes \lambda(g)$ be a finite sum with $x_{g} \in S_{\infty}$. Write

$$
x=\operatorname{id}_{S_{\infty}} \otimes P(x)+\operatorname{id}_{S_{\infty}} \otimes Q(x)=\sum_{k} x_{k} \otimes \lambda\left(g_{k}\right)+\operatorname{id}_{S_{\infty}} \otimes Q(x) .
$$

Then using the argument yielding the first inequality of (6.4), we get

$$
\left\|\left(x_{k}\right)\right\|_{C R_{\infty}\left[S_{\infty}\right]} \leq\|x\|_{\infty} .
$$

Together with a duality argument as above, this inequality implies the upper estimate of (6.5).

Combining (6.4) and (6.6), we get

$$
\|P(x)\|_{\infty} \leq 2\|x\|_{\infty} .
$$

This implies that $P$ extends to a completely bounded normal projection on $V N(\mathbb{F})$. Indeed, by the density of all finite sums $\sum_{g \in \mathbb{F}} x_{g} \otimes \lambda(g), x_{g} \in S_{1}$, in $L_{1}\left(B\left(\ell_{2}\right) \bar{\otimes} V N(\mathbb{F})\right)$, we find that (6.5) shows that $P$ admits an extension on $L_{1}(V N(\mathbb{F}))$ which is completely bounded with cb-norm $\leq 2$. Taking adjoint, we obtain the desired completely bounded normal extension of $P$ on $V N(\mathbb{F})$. The complete boundedness of $P$ on $L_{p}(V N(\mathbb{F}))$ is then proved by interpolation.

6.5 Semicircular systems. Let $H$ be a complex Hilbert space. The associated free (or full) Fock space is defined by

$$
\mathcal{F}(H)=\bigoplus_{n \geq 0} H^{\otimes n},
$$

where $H^{\otimes 0}=\mathbb{C} \mathbb{1}$ (11 being a unit vector, called vacuum), and $H^{\otimes n}$ is the n-th Hilbertian tensor power of $H$ for $n \geq 1$. The (left) creator associated with a vector $\xi \in H$ is the operator on $\mathcal{F}(H)$ uniquely determined by

$$
c(\xi) \eta_{1} \otimes \cdots \otimes \eta_{n}=\xi \otimes \eta_{1} \otimes \cdots \otimes \eta_{n}
$$


for any $\eta_{1}, \ldots, \eta_{n} \in H$. Here $\eta_{1} \otimes \cdots \otimes \eta_{n}$ is understood as the vacuum $\mathbb{1}$ if $n=0$. It is clear that $c(\xi)$ is bounded and $\|c(\xi)\|=\|\xi\|$. The adjoint of $c(\xi)$ is given by

$$
c(\xi)^{*} \eta_{1} \otimes \cdots \otimes \eta_{n}=\left\langle\eta_{1}, \xi\right\rangle \eta_{2} \otimes \cdots \otimes \eta_{n}
$$

for any $\eta_{1}, \ldots, \eta_{n} \in H$ with $n \geq 1$ and $c(\xi)^{*} \mathbb{1}=0$. This is the annihilator associated with $\xi$ and is denoted by $a(\xi)$. Note that the map $\xi \mapsto c(\xi)$ is linear, while $\xi \mapsto a(\xi)$ is anti-linear. We have the following free commutation relation:

$$
a(\eta) c(\xi)=\langle\xi, \eta\rangle 1, \quad \forall \xi, \eta \in H .
$$

Now assume that $H$ is the complexification of a real Hilbert space $H_{\mathbb{R}}$. The vectors $H_{\mathbb{R}}$ are called real. Let $\xi \in H$ be real and define

$$
s(\xi)=c(\xi)+a(\xi) .
$$

$s(\xi)$ is a semicircular element in Voiculescu's sense. We will also call it a free Gaussian variable. Note that the map $\xi \mapsto s(\xi)$ is real linear from $H_{\mathbb{R}}$ into $B(\mathcal{F}(H))$. Then the free von Neumann algebra $\Gamma(H)$ associated with $H$ is the von Neumann subalgebra of $B(\mathcal{F}(H))$ generated by all $s(\xi)$ with real $\xi \in H$ :

$$
\Gamma_{0}(H)=\left\{s(\xi): \xi \in H_{\mathbb{R}}\right\}^{\prime \prime} \subset B(\mathcal{F}(H)) .
$$

The vector state $\tau_{0}$ defined by the vacuum, $x \mapsto\langle x \mathbb{1}, \mathbb{1}\rangle$ is faithful and tracial on $\Gamma_{0}(H)$. We refer to [VDN] for more information.

Let us mention some basic properties of free Gaussian variables. Let $\xi \in H$ be a unit real vector. By definition, $s(\xi)$ is selfadjoint; its spectrum is $[-2,2]$ and the corresponding measure induced by $\tau_{0}$ is the so-called Wigner measure

$$
d \mu(t)=\frac{1}{2 \pi} \sqrt{4-t^{2}} d t .
$$

Thus for any $1 \leq p<\infty$

$$
\|s(\xi)\|_{p}=\left[\int_{-2}^{2}|t|^{p} \sqrt{4-t^{2}} \frac{d t}{2 \pi}\right]^{1 / p} .
$$

Therefore,

$$
\|s(\xi)\|_{\infty}=2, \quad\|s(\xi)\|_{2}=1, \quad\|s(\xi)\|_{1}=\frac{8}{3 \pi} .
$$

Now let $\left(\xi_{k}\right)$ be an orthonormal sequence of real vectors of $H .\left(s\left(\xi_{k}\right)\right)$ is then called a standard semicircular system. Like the classical standard Gaussian variables, $\left(s\left(\xi_{k}\right)\right)$ has the following invariance property. Let $\left(\alpha_{k}\right) \subset \mathbb{R}$ be such that $\sum_{k}\left|\alpha_{k}\right|^{2}=1$. Then $\sum_{n} \alpha_{k} s\left(\xi_{k}\right)$ (convergence in the strong operator topology) is again a free Gaussian variable, i.e., $s(\xi)$ with $\xi=\sum_{k} \alpha_{k} \xi_{k}$. More generally, if $s\left(\xi_{1}\right), \ldots, s\left(\xi_{n}\right)$ is a standard semi-circular system and if $u=\left(u_{j k}\right)$ is an orthogonal $n \times n$ matrix, then

$$
\left(\sum_{k=1}^{n} u_{j k} s\left(\xi_{k}\right)\right)_{1 \leq j \leq n}
$$

is still a standard semi-circular system. Indeed,

$$
\sum_{k=1}^{n} u_{j k} s\left(\xi_{k}\right)=s\left(\sum_{k=1}^{n} u_{j k} \xi_{k}\right)=s\left(\eta_{j}\right),
$$

where $\eta_{j}=\sum_{k} u_{j k} \xi_{k}$. The $\eta_{j}$ form an orthonormal family. This orthogonal invariance implies that if $\left(s\left(\xi_{k}\right)\right)$ is a standard semicircular system, then for $\left(\alpha_{k}\right) \subset \mathbb{R}$ such that $\sum_{k}\left|\alpha_{k}\right|^{2}<\infty$ and any $1 \leq p \leq \infty$

$$
\left\|\sum_{k \geq 1} \alpha_{k} s\left(\xi_{k}\right)\right\|_{p}=\left(\sum_{k \geq 1}\left|\alpha_{k}\right|^{2}\right)^{1 / 2}\|s(\xi)\|_{p} .
$$


In particular, $\left(s\left(\xi_{k}\right)\right)$ is an orthonormal sequence in $L_{2}(\Gamma(H))$.

For simplicity we now assume that $H$ is infinite dimensional and separable and put $\Gamma_{0}=\Gamma_{0}(H)$. Let $\left(e_{k}\right)$ be an orthonormal basis of $H$ and set $s_{k}=s\left(e_{k}\right)$. Thus $\left(s_{k}\right)$ is a semicircular sequence and $\Gamma_{0}$ is generated by the $s_{k}$. The following inequality for semicircular systems comes from [P2].

Theorem 6.9 Let $1 \leq p \leq \infty$ and $\left(x_{k}\right)$ be a finite sequence in $S_{p}$. Then

$$
\left\|\sum_{k} x_{k} \otimes s_{k}\right\|_{L_{p}\left(B\left(\ell_{2}\right) \bar{\otimes} \Gamma_{0}\right)} \sim_{c}\left\|\left(x_{k}\right)\right\|_{C R_{p}\left[S_{p}\right]} .
$$

Moreover, the closed subspace of $L_{p}\left(\Gamma_{0}\right)$ generated by the $s_{k}$ is completely complemented in $L_{p}\left(\Gamma_{0}\right)$ with constant 2.

Proof. This proof is similar to that of Theorem 6.8. Again, the case $1<p<\infty$ will be proved later. By the free commutation relation (6.7), we have

$$
\left\|\sum_{k} x_{k} \otimes c\left(e_{k}\right)\right\|_{\infty}^{2}=\left\|\left(\sum_{k} x_{k} \otimes c\left(e_{k}\right)\right)^{*}\left(\sum_{k} x_{k} \otimes c\left(e_{k}\right)\right)\right\|_{\infty}=\left\|\sum_{k} x_{k}^{*} x_{k}\right\|_{\infty} .
$$

Similarly,

$$
\left\|\sum_{k} x_{k} \otimes a\left(e_{k}\right)\right\|_{\infty}^{2}=\left\|\sum_{k} x_{k} x_{k}^{*}\right\|_{\infty}
$$

It follows that

$$
\left\|\sum_{k} x_{k} \otimes s_{k}\right\|_{\infty} \leq\left\|\left(\sum_{k} x_{k}^{*} x_{k}\right)^{\frac{1}{2}}\right\|_{\infty}+\left\|\left(\sum_{k} x_{k} x_{k}^{*}\right)^{\frac{1}{2}}\right\|_{\infty} .
$$

For the lower estimate, take a unit vector $\xi \in \ell_{2}$. Then

$$
\begin{aligned}
\left\|\sum_{k} x_{k} \otimes s_{k}\right\|_{\infty}^{2} & \geq\left\langle\sum_{k} x_{k} \otimes s_{k}(\xi \otimes \mathbb{1}), \sum_{k} x_{k} \otimes s_{k}(\xi \otimes \mathbb{1})\right\rangle \\
& =\left\langle\sum_{k} x_{k}(\xi) \otimes e_{k}, \sum_{k} x_{k}(\xi) \otimes e_{k}\right\rangle \\
& =\left\langle\sum_{k} x_{k}^{*} x_{k}(\xi), \xi\right\rangle ;
\end{aligned}
$$

whence

$$
\left\|\left(\sum_{k} x_{k}^{*} x_{k}\right)^{\frac{1}{2}}\right\|_{\infty} \leq\left\|\sum_{k} x_{k} \otimes s_{k}\right\|_{\infty}
$$

Similarly,

$$
\left\|\left(\sum_{k} x_{k} x_{k}^{*}\right)^{\frac{1}{2}}\right\|_{\infty} \leq\left\|\sum_{k} x_{k} \otimes s_{k}\right\|_{\infty} .
$$

Thus the lower estimate of (6.8) for $p=\infty$ follows.

(6.8) for $p=1$ and the complementation assertion are proved in the same way as the corresponding assertions of Theorem 6.8. We omit the details.

Remark 6.10 The free Gaussian variables in the theorem above can be replaced by BożejkoSpeicher's $q$-Gaussians for $-1<q<1$ (see [BKS] and [BS]). This inequality remains also true for the case $q=-1$, for which the corresponding Gaussians become the so-called Fermions. Note that the case $q=1$ corresponds to the classical Gaussian case. In strong contrast with the case $-1<q<1$, the analogue for $q= \pm 1$ of Theorem 6.9 fails for $p=\infty$. 
6.6 Complete unconditionality. Noncommutative Khintchine inequalities are closely related to complete unconditionality. Let $M$ be a von Neumann algebra equipped with a normal faithful tracial state $\tau$. Let $\left(a_{k}\right)$ be a sequence in $L_{p}(M), 1 \leq p \leq \infty$. Assume that the noncommutative Khintchine inequality holds for $\left(a_{k}\right)$ : for any finite sequence $\left(x_{k}\right)$ in $S_{p}$

$$
\left\|\sum_{k} x_{k} \otimes a_{k}\right\|_{L_{p}\left(B\left(\ell_{2}\right) \bar{\otimes} M\right)} \sim\left\|\left(x_{k}\right)\right\|_{C R_{p}\left[S_{p}\right]} .
$$

It then follows that the subspace $E$ generated by the $a_{k}$ in $L_{p}(M)$ is completely isomorphic to $C R_{p}$ (see Remark 6.6). Since the canonical basis of $C R_{p}$ is completely unconditional, so is the sequence $\left(a_{k}\right)$. Namely, there exists a constant $\lambda$ such that

$$
\left\|\sum_{k} \varepsilon_{k} x_{k} \otimes a_{k}\right\|_{p} \leq \lambda\left\|\sum_{k} x_{k} \otimes a_{k}\right\|_{p}
$$

for any finite sequence $\left(x_{k}\right) \subset S_{p}$ and any $\varepsilon_{k}= \pm 1$. This property can be rephrased as follows. Given any sequence $\left(\varepsilon_{k}\right)$ of signs the map $\sum_{k} \alpha_{k} a_{k} \mapsto \sum_{k} \varepsilon_{k} \alpha_{k} a_{k}$ on $E$ is c.b. with cb-norm $\leq \lambda$. Therefore, if the noncommutative Khintchine inequality holds for $\left(a_{k}\right)$, then $\left(a_{k}\right)$ is a completely unconditional basic sequence. The converse is also true with some additional mild conditions for $p<\infty$. We consider here only the case $1 \leq p<2$. The following result is proved independently by the author and Junge/Oikhberg [JO]. The latter paper contains more results of the same type.

Theorem 6.11 Let $M$ be a von Neumann algebra equipped with a normal faithful tracial state $\tau$. Let $1 \leq p<2$ and $\left(a_{k}\right)_{k \geq 1} \subset L_{p}(M)$ be a completely unconditional basic sequence with constant $\lambda$. Assume that

$$
\delta=\inf _{k}\left\|a_{k}\right\|_{p}>0 \quad \text { and } \quad \Delta=\sup _{k}\left\|a_{k}\right\|_{2}<\infty .
$$

Then the noncommutative Khintchine inequality holds for $\left(a_{k}\right)$ with relevant constants depending only on $\lambda, \delta$ and $\Delta$. More precisely, for any finite sequence $\left(x_{k}\right) \subset S_{p}$

$$
c \delta \lambda^{-1}\left\|\left(x_{k}\right)\right\|_{C R_{p}\left[S_{p}\right]} \leq\left\|\sum_{k} x_{k} \otimes a_{k}\right\|_{p} \leq \Delta \lambda\left\|\left(x_{k}\right)\right\|_{C R_{p}\left[S_{p}\right]},
$$

where $c$ is an absolute positive constant.

Proof. Let $\left(x_{k}\right) \subset S_{p}$ be a finite sequence. We then have (6.9). Averaging the left hand side of (6.9) over the $\varepsilon_{n}$ and using the noncommutative Khintchine inequality in Theorem 6.5, we get

$$
\inf \left\{\left\|\left(\sum_{k} Y_{k}^{*} Y_{k}\right)^{1 / 2}\right\|_{p}+\left\|\left(\sum_{k} Z_{k} Z_{k}^{*}\right)^{1 / 2}\right\|_{p}\right\} \leq c \lambda\left\|\sum_{k} x_{k} \otimes a_{k}\right\|_{p}
$$

where the infimum runs over all decompositions $x_{k} \otimes a_{k}=Y_{k}+Z_{k}$ with $Y_{k}$ and $Z_{k}$ in $L_{p}\left(B\left(\ell_{2}\right) \bar{\otimes} M\right)$. Fix such a decomposition $x_{k} \otimes a_{k}=Y_{k}+Z_{k}$. Now choose $b_{k} \in L_{p^{\prime}}(M)$ such that

$$
\tau\left(a_{k} b_{k}\right)=1 \quad \text { and } \quad\left\|b_{k}\right\|_{p^{\prime}}=\left\|a_{k}\right\|_{p}^{-1}
$$

Then

$$
x_{k}=\mathrm{id} \otimes \tau\left(\left(x_{k} \otimes a_{k}\right)\left(1 \otimes b_{k}\right)\right)=\mathrm{id} \otimes \tau\left(Y_{k}\left(1 \otimes b_{k}\right)\right)+\mathrm{id} \otimes \tau\left(Z_{k}\left(1 \otimes b_{k}\right)\right) \stackrel{\text { def }}{=} y_{k}+z_{k} .
$$

Note that id $\otimes \tau$ is the natural conditional expectation from $B\left(\ell_{2}\right) \bar{\otimes} M$ onto $B\left(\ell_{2}\right)\left(B\left(\ell_{2}\right)\right.$ being viewed as a von Neumann subalgebra of $B\left(\ell_{2}\right) \bar{\otimes} M$ via $\left.x \leftrightarrow x \otimes 1\right)$. Also note that $y_{k}$ and $z_{k}$ belong to $S_{p}$. We need to majorize $\left\|\left(\sum y_{k}^{*} y_{k}\right)^{1 / 2}\right\|_{p}\left(\operatorname{resp}\right.$. $\left.\left\|\left(\sum z_{k} z_{k}^{*}\right)^{1 / 2}\right\|_{p}\right)$ by $\left\|\left(\sum Y_{k}^{*} Y_{k}\right)^{1 / 2}\right\|_{p}$ 
(resp. $\left.\left\|\left(\sum Z_{k} Z_{k}^{*}\right)^{1 / 2}\right\|_{p}\right)$. To this end, let $\left(u_{k}\right) \subset S_{p^{\prime}}$ be such that $\left\|\left(\sum_{k} u_{k} u_{k}^{*}\right)^{1 / 2}\right\|_{p^{\prime}} \leq 1$. By the Hölder inequality in Proposition 6.1

$$
\begin{aligned}
\left|\sum_{k} \operatorname{Tr}\left(y_{k} u_{k}\right)\right| & =\left|\sum_{k} \operatorname{Tr}\left[\operatorname{id} \otimes \tau\left(Y_{k}\left(1 \otimes b_{k}\right)\right) u_{k}\right]\right|=\left|\sum_{k} \operatorname{Tr} \otimes \tau\left[Y_{k}\left(u_{k} \otimes b_{k}\right)\right]\right| \\
& \leq\left\|\left(\sum_{k} u_{k} u_{k}^{*} \otimes b_{k} b_{k}^{*}\right)^{1 / 2}\right\|_{p^{\prime}}\left\|\left(\sum_{k} Y_{k}^{*} Y_{k}\right)^{1 / 2}\right\|_{p} .
\end{aligned}
$$

We claim that

$$
\left\|\left(\sum_{k} u_{k} u_{k}^{*} \otimes b_{k} b_{k}^{*}\right)^{1 / 2}\right\|_{p^{\prime}} \leq \sup _{k}\left\|b_{k}\right\|_{p^{\prime}}\left\|\left(\sum_{k} u_{k} u_{k}^{*}\right)^{1 / 2}\right\|_{p^{\prime}} .
$$

Indeed, this is obvious for $p^{\prime}=2$ and $p^{\prime}=\infty$. Then complex interpolation yields the case $2<p^{\prime}<\infty$. Combining the preceding inequalities, we find

$$
\begin{aligned}
\left|\sum_{k} \operatorname{Tr}\left(y_{k} u_{k}\right)\right| & \leq \sup _{k}\left\|b_{k}\right\|_{p^{\prime}}\left\|\left(\sum_{k} u_{k} u_{k}^{*}\right)^{1 / 2}\right\|_{p^{\prime}}\left\|\left(\sum_{k} Y_{k}^{*} Y_{k}\right)^{1 / 2}\right\|_{p} \\
& \leq \delta^{-1}\left\|\left(\sum_{k} Y_{k}^{*} Y_{k}\right)^{1 / 2}\right\|_{p} .
\end{aligned}
$$

Thus taking the supremum over all $\left(u_{k}\right)$, we get

$$
\left\|\left(\sum_{k} y_{k}^{*} y_{k}\right)^{1 / 2}\right\|_{p} \leq \delta^{-1}\left\|\left(\sum_{k} Y_{k}^{*} Y_{k}\right)^{1 / 2}\right\|_{p}
$$

Similarly,

$$
\left\|\left(\sum_{k} z_{k} z_{k}^{*}\right)^{1 / 2}\right\|_{p} \leq \delta^{-1}\left\|\left(\sum_{k} Z_{k} Z_{k}^{*}\right)^{1 / 2}\right\|_{p}
$$

Therefore, we deduce

$$
\left\|\left(x_{k}\right)\right\|_{C R_{p}\left[S_{p}\right]} \leq c \delta^{-1} \lambda\left\|\sum_{k} x_{k} \otimes a_{k}\right\|_{p} .
$$

To prove the upper estimate we use again the complete unconditionality of $\left(a_{k}\right)$ and the noncommutative Khintchine inequality. Then we have

$$
\left\|\sum_{k} x_{k} \otimes a_{k}\right\|_{p} \leq \lambda \inf _{x_{k}=y_{k}+z_{k}}\left\{\left\|\left(\sum_{k} y_{k}^{*} y_{k} \otimes a_{k}^{*} a_{k}\right)^{1 / 2}\right\|_{p}+\left\|\left(\sum_{k} z_{k} z_{k}^{*} \otimes a_{k} a_{k}^{*}\right)^{1 / 2}\right\|_{p}\right\} .
$$

Now our task is to remove $a_{k}^{*} a_{k}$ and $a_{k} a_{k}^{*}$ from the terms on the right. To this end we use the natural conditional expectation $\mathbb{E}$ from $B\left(\ell_{2}\right) \bar{\otimes} M$ onto $B\left(\ell_{2}\right)$, already mentioned earlier. $\mathbb{E}$ is determined by $\mathbb{E}(x \otimes u)=\tau(u) x \otimes 1 \sim \tau(u) x$ for $x \in B\left(\ell_{2}\right)$ and $u \in M$, i.e., $\mathbb{E}=$ id $\otimes \tau$. $\mathbb{E}$ is normal and faithful. As usual, $\mathbb{E}$ extends to a contractive projection on $L_{q}\left(B\left(\ell_{2}\right) \bar{\otimes} M\right)$ for every $1 \leq q<\infty$. For our purpose here we need to consider the case $q \leq 1$. We claim that if $X$ is a positive operator in $L_{q}\left(B\left(\ell_{2}\right) \bar{\otimes} M\right) \cap L_{1}\left(B\left(\ell_{2}\right) \bar{\otimes} M\right)$ with $q \leq 1$, then

$$
\|X\|_{q} \leq\|\mathbb{E}(X)\|_{q} .
$$

This is a consequence of the operator concavity of the map $X \mapsto X^{q}$ for $0<q \leq 1$. Indeed, using Stinespring's dilation theorem and Hansen's inequality [Ha], we deduce that $(\mathbb{E}(X))^{q} \geq \mathbb{E}\left(X^{q}\right)$ for any positive $X \in B\left(\ell_{2}\right) \bar{\otimes} M$. This clearly implies (6.11). 
Return back to our task. Using (6.11) with $q=p / 2$ (recalling that $1 \leq p<2$ ), we deduce that

$$
\begin{aligned}
\left\|\left(\sum_{k} y_{k}^{*} y_{k} \otimes a_{k}^{*} a_{k}\right)^{1 / 2}\right\|_{p}^{2} & =\left\|\sum_{k} y_{k}^{*} y_{k} \otimes a_{k}^{*} a_{k}\right\|_{p / 2} \\
& \leq\left\|\mathbb{E}\left[\sum_{k} y_{k}^{*} y_{k} \otimes a_{k}^{*} a_{k}\right]\right\|_{p / 2} \\
& =\left\|\sum_{k} y_{k}^{*} y_{k} \otimes \tau\left(a_{k}^{*} a_{k}\right) 1\right\|_{p / 2} \\
& \leq \sup _{k}\left\|a_{k}\right\|_{2}^{2}\left\|\left(\sum_{k} y_{k}^{*} y_{k}\right)^{1 / 2}\right\|_{p}^{2} .
\end{aligned}
$$

Therefore,

$$
\left\|\left(\sum_{k} y_{k}^{*} y_{k} \otimes a_{k}^{*} a_{k}\right)^{1 / 2}\right\|_{p} \leq \Delta\left\|\left(\sum_{k} y_{k}^{*} y_{k}\right)^{1 / 2}\right\|_{p} .
$$

A similar inequality holds for another term involving the $z_{k}$. It thus follows that

$$
\left\|\sum_{k} x_{k} \otimes a_{k}\right\|_{p} \leq \Delta \lambda\left\|\left(x_{k}\right)\right\|_{C R_{p}\left[S_{p}\right]} .
$$

This is the desired upper estimate, and so the proof of the theorem is complete.

End of the proofs of Theorems 6.8 and 6.9. The $\lambda\left(g_{k}\right)$ are unitary, so $\left\|\lambda\left(g_{k}\right)\right\|_{p}=1$ for any $1 \leq p \leq \infty$. On the other hand, for any sequence $\left(\varepsilon_{k}\right)$ of sings, there exists a unique representation $\pi$ of $V N(\mathbb{F})$ determined by $\pi\left(\lambda\left(g_{k}\right)\right)=\varepsilon_{k} \lambda\left(g_{k}\right)$. Moreover, $\pi$ is trace preserving: $\tau_{\mathbb{F}} \circ \pi=\tau_{\mathbb{F}}$. It follows that $\pi$ extends to a complete isometry on $L_{p}(V N(\mathbb{F}))$ for every $1 \leq p \leq \infty$. Therefore, for any finite sequence $\left(x_{k}\right) \subset S_{p}$ we have

$$
\left\|\sum_{k} \varepsilon_{k} x_{k} \otimes \lambda\left(g_{k}\right)\right\|_{p}=\left\|\sum_{k} x_{k} \otimes \lambda\left(g_{k}\right)\right\|_{p} .
$$

Thus the sequence $\left(\lambda\left(g_{k}\right)\right)$ is completely unconditional with constant 1 . Then Theorem 6.11 implies (6.3) for $1 \leq p<2$. The case $p=2$ is trivial. The case $2<p<\infty$ is obtained by duality and using the complementation property in Theorem 6.11.

The proof of Theorem 6.9 is similar. This time to get the representation $\pi$ of $\Gamma_{0}$ such that $\pi\left(s_{k}\right)=\varepsilon_{k} s_{k}$, we have to use second quantization (see [VDN]).

Remark 6.12 There exist many examples satisfying the assumption of Theorem 6.11. This is the case of a sequence of $q$-Gaussians mentioned in Remark 6.10. In particular, for $q=-1$, we get the noncommutative Khintchine inequality for a sequence of Fermions.

6.7 Generalized circular systems. Remind that we wish to embed $O H$ into a noncommutative $L_{1}$-space by using a certain noncommutative Khintchine type inequality. Namely, we have to prove that $O H$ is completely isomorphic to the closed subspace generated by a sequence of random variables in an $L_{1}(M)$ for a von Neumann algebra $M$. Such a sequence cannot satisfy the assumption of Theorem 6.11 for $O H$ is not completely isomorphic to $C R_{1}$. In fact, Pisier [P5] showed that $O H$ cannot completely embed into an $L_{1}(M)$ with $M$ semifinite (i.e., of type I or II). This explains why we are forced to seek for Khintchine type inequalities for random variables in a non tracial probability space, i.e., the underlying von Neumann algebra is of type III. We give below only one example of this kind.

Fix an infinite dimensional separable Hilbert space $H$ as in subsection 6.5. Let $\left\{e_{ \pm k}\right\}_{k \geq 1}$ be an orthonormal basis of $H$. We also fix a sequence $\left\{\lambda_{k}\right\}_{k \geq 1}$ of positive numbers. Let

$$
g_{k}=c\left(e_{k}\right)+\sqrt{\lambda_{k}} a\left(e_{-k}\right) .
$$


$\left(g_{k}\right)_{k \geq 1}$ is a generalized circular system in Shlyakhtenko's sense [S]. Let $\Gamma$ be the von Neumann algebra on the full Fock space $\mathcal{F}(H)$ generated by the $g_{k}$. Let $\rho$ be the vector state on $\Gamma$ determined by the vacuum $\mathbb{1}$. Then $\rho$ is faithful on $\Gamma$. By the identification of $L_{1}(\Gamma)$ with the predual $\Gamma_{*}, \rho$ is a positive unit element of $L_{1}(\Gamma)$, so for any $1 \leq p \leq \infty, \rho^{1 / p}$ is a positive unit element of $L_{p}(\Gamma)$, and thus $g_{k} \rho^{1 / p} \in L_{p}(\Gamma)$ for any $k$.

Shlyakhtenko proved that the algebra $\Gamma$ is a type $\mathrm{III}_{\lambda}$ factor $(0<\lambda \leq 1)$ if not all $\lambda_{k}$ are equal to $1 . \Gamma$ is not hyperfinite. Recall that $\Gamma$ is the free analogue of the classical Araki-Woods quasi-free CAR factors. The latter factors are hyperfinite type $\mathrm{III}_{\lambda}$.

The following is the noncommutative Khintchine type inequalities for generalized circular systems, proved in [PS] for $p=\infty$ and in [X2] for $p<\infty$.

Theorem 6.13 Let $\left(x_{n}\right)$ be a finite sequence in $S_{p}, 1 \leq p \leq \infty$.

i) If $p \geq 2$,

$$
\left\|\sum_{k} x_{k} \otimes g_{k} \rho^{\frac{1}{p}}\right\|_{L_{p}\left(B\left(\ell_{2}\right) \bar{\otimes} \Gamma\right)} \sim_{c} \max \left\{\left\|\left(\sum_{k} x_{k}^{*} x_{k}\right)^{\frac{1}{2}}\right\|_{p},\left\|\left(\sum_{k} \lambda_{k}^{1-\frac{2}{p}} x_{k} x_{k}^{*}\right)^{\frac{1}{2}}\right\|_{p}\right\} .
$$

ii) If $p<2$,

$$
\left\|\sum_{k} x_{k} \otimes g_{k} \rho^{\frac{1}{p}}\right\|_{L_{p}\left(B\left(\ell_{2}\right) \bar{\otimes} \Gamma\right)} \sim_{c} \inf \left\{\left\|\left(\sum_{k} y_{k}^{*} y_{k}\right)^{\frac{1}{2}}\right\|_{p}+\left\|\left(\sum_{k} \lambda_{k}^{1-\frac{2}{p}} z_{k} z_{k}^{*}\right)^{\frac{1}{2}}\right\|_{p}\right\},
$$

where the infimum runs over all decompositions $x_{k}=y_{k}+z_{k}$ in $S_{p}$.

iii) Let $\mathcal{G}_{p}$ be the closed subspace of $L_{p}(\Gamma)$ generated by $\left\{g_{k} \rho^{\frac{1}{p}}\right\}_{k \geq 1}$. Then $\mathcal{G}_{p}$ is completely complemented in $L_{p}(\Gamma)$ with constant 2.

Proof. We prove only the cases $p=\infty, 1$ and the complementation assertion. The proof of i) for $p=\infty$ is the same as that of (6.4). Let us prove iii), which will imply ii) for $p=1$ by duality. As in the semicircular case, consider the projection $P$ from the algebra of polynomials on the $g_{k}$ onto the linear span of the $g_{k}$. Let $Q=I-P$. It is easy to see that $P(x)$ and $Q(x)$ are orthogonal relative to both scalar products $(a, b) \mapsto \rho\left(b^{*} a\right)$ and $(a, b) \mapsto \rho\left(a b^{*}\right)$. Now let $x$ be a polynomial on the $g_{k}$ with coefficients in $S_{\infty}$. Write $x$ as

$$
x=\operatorname{id}_{S_{\infty}} \otimes P(x)+\operatorname{id}_{S_{\infty}} \otimes Q(x)=\sum_{k} a_{k} \otimes g_{k}+\operatorname{id}_{S_{\infty}} \otimes Q(x) .
$$

Then using the argument yielding the first inequality of (6.4), we get

$$
\max \left\{\left\|\left(\sum_{k} a_{k}^{*} a_{k}\right)^{\frac{1}{2}}\right\|_{\infty},\left\|\left(\sum_{k} \lambda_{k} a_{k} a_{k}^{*}\right)^{\frac{1}{2}}\right\|_{\infty}\right\} \leq\|x\|_{\infty} .
$$

Therefore, using i) in the case $p=\infty$, we deduce that $P$ is completely bounded. This implies, in turn, ii) for $p=1$ by duality. Let us also note that $P$ extends to a completely bounded normal projection on $\Gamma$. Indeed, by the density of $\left\{x \rho: x\right.$ polynomial on the $\left.g_{k}\right\}$ in $L_{1}(\Gamma)$, we find that ii) in the case $p=1$ shows that $P$ admits a pre-adjoint on $L_{1}(\Gamma)$ which is completely bounded. Finally, by interpolation we obtain iii) for $1<p<\infty$.

Remark 6.14 In the spirit of Remark 6.6, Theorem 6.11 can be reformulated as a complete isomorphism between the subspace $\mathcal{G}_{p}$ and a weighted version of $C R_{p}$. Given a sequence $\left(\mu_{k}\right)$ of positive numbers we denote by $C\left(\left(\mu_{k}\right)\right)$ the weighted $\ell_{2}$-space $\ell_{2}\left(\left(\mu_{k}\right)\right)$ equipped with the column Hilbert space structure. More generally, for any $p \geq 1$ we define $C_{p}\left(\left(\mu_{k}\right)\right)$ to be the weighted 
version of $C_{p}$. Note that $C_{p}\left(\left(\mu_{k}\right)\right)$ is completely isometric to $C_{p}$ and its operator space structure is determined as follows: for any finite sequence $\left(x_{k}\right) \subset S_{p}$

$$
\left\|\sum_{k} x_{k} \otimes e_{k}\right\|_{S_{p}\left[C_{p}\left(\left(\mu_{k}\right)\right)\right]}=\left\|\left(\sum_{k} \mu_{k} x_{k}^{*} x_{k}\right)^{\frac{1}{2}}\right\|_{S_{p}} .
$$

Similarly, we define the weighted $p$-row space $R_{p}\left(\left(\mu_{k}\right)\right)$. Then Theorem 6.11 implies that $\mathcal{G}_{p}$ is completely isomorphic to $C_{p} \cap R_{p}\left(\left(\mu_{k}\right)\right)$ for $p \geq 2$ and to $C_{p}+R_{p}\left(\left(\mu_{k}\right)\right)$ for $p<2$, where $\mu_{k}=\lambda_{k}^{1-2 / p}$.

Remark 6.15 Theorem 6.11 admits a Fermionic analogue. In this case the corresponding algebra is an Araki-Woods hyperfinite type III factor. The resulting inequality holds only for $p<\infty$ and the relevant constants depend only on $p$ and blow up as $p \rightarrow \infty$. We refer to [J3] and [X1] for more information (see also $[\mathrm{HM}]$ for an alternate approach for the case $p=1$ ).

\section{Exercices:}

1) Prove the classical Khintchine inequality (6.1). (Consider first the case of an even integer $p$.)

2) Prove that there exists no constant $c_{p}$ depending on $p$ such that

$$
\left\|\sum_{k} x_{k} \varepsilon_{n}\right\|_{L_{p}\left(\Omega ; S_{p}\right)} \leq c_{p}\left\|\left(x_{k}\right)\right\|_{C_{p}\left[S_{p}\right]} \text { if } p>2
$$

or

$$
\left\|\left(x_{k}\right)\right\|_{C_{p}\left[S_{p}\right]} \leq c_{p}\left\|\sum_{k} x_{k} \varepsilon_{n}\right\|_{L_{p}\left(\Omega ; S_{p}\right)} \quad \text { if } p<2
$$

holds for all finite sequences $\left(x_{k}\right) \subset S_{p}$.

3) Prove Remark 6.7.

4) Let $G$ be a discrete group. Prove that the vector state $\tau_{G}$ determined by $\delta_{e}$ is faithful and tracial on $V N(G)$.

5) Prove that the vector state $\tau_{0}$ determined by the vacuum $\mathbb{1}$ is faithful and tracial on the free von Neumann algebra $\Gamma_{0}(H)$.

\section{Embedding of $O H$ into noncommutative $L_{1}$}

Now we use the Khintchine inequality for generalized circular systems to embed $O H$ completely isomorphically into a noncommutative $L_{1}$-space, i.e., to show that $O H$ is completely isomorphic to a subspace of a noncommutative $L_{1}$. This is a remarkable theorem of Junge [J2]. The following representation of $O H$ as a quotient of a subspace of $C \oplus R$ will be crucial.

Theorem 7.1 $\mathrm{OH}$ is completely isometric to a quotient of a subspace of $C \oplus_{\infty} R$.

The direct sum in the $\ell_{\infty}$-sense can be replaced by a direct sum in the $\ell_{1}$-sense at the price of a constant 2 . Since the complete embeddings in the sequel are only completely isomorphic, we will forget the index $\infty$ or 1 in all direct sums. The preceding theorem is Exercice 7.8 of [P3]. The proof there gives the following more precise statement.

Theorem 7.2 There exists an injective positive selfadjoint (unbounded) operator $\Delta: C \rightarrow R$ such that $O H$ is completely isometric to a quotient of $G(\Delta)$, where $G(\Delta)$ is the graph of $\Delta$

$$
G(\Delta)=\{(h, \Delta h): h \in D(\Delta)\} \subset C \oplus R,
$$

considered as a subspace of $C \oplus R$. 
Recall that both $C$ and $R$ are isometric to $\ell_{2}$ as Banach spaces. Thus $\Delta$ is an operator on $\ell_{2}$ with domain $D(\Delta)$. Since $\Delta$ is positive and injective, its range is also dense. Passing to duality, we see that $O H^{*}$ is completely isometric to a subspace of $G(\Delta)^{*}$. Since $\overline{O H^{*}}=O H$ completely isometrically, embedding $O H$ into an $L_{1}$ is reduced to embedding $G(\Delta)^{*}$ into an $L_{1}$.

To see why Khintchine type inequalities can help us for such a matter, let us describe the operator space structure of $G(\Delta)$ :

$$
G(\Delta)=\{(h, \Delta(h)): h \in D(\Delta)\} \subset C \oplus_{\infty} R .
$$

Let $x_{k} \in S_{\infty}$ and $h_{k} \in D(\Delta)$. Let $x=\sum_{k} x_{k} \otimes\left(h_{k}, \Delta\left(h_{k}\right)\right) \in S_{\infty} \otimes_{\min } G(\Delta)$. Then

$$
\|x\|_{S_{\infty} \otimes_{\min } G(\Delta)}=\max \left\{\left\|\sum_{k, j}\left\langle h_{k}, h_{j}\right\rangle x_{j}^{*} x_{k}\right\|_{S_{\infty}}^{1 / 2},\left\|\sum_{k, j}\left\langle\Delta\left(h_{k}\right), \Delta\left(h_{j}\right)\right\rangle x_{k} x_{j}^{*}\right\|_{S_{\infty}}^{1 / 2}\right\} .
$$

This is a continuous version of the space (for $p=\infty$ ) introduced in Remark 6.14. To simplify our discussion and without loss of generality by a simple argument of approximation, we may assume that $\Delta$ has pure point spectrum, i.e., $\ell_{2}$ has an orthonormal basis $\left(e_{k}\right)$ of eigenvectors of $\Delta$. Let $\left(\mu_{k}\right)$ be the associated eigenvalues: $\Delta e_{k}=\mu_{k} e_{k}$. It then follows that $G(\Delta)$ coincides (completely isometrically) with the diagonal subspace $C \cap R\left(\left(\lambda_{k}\right)\right)$ of $C \oplus_{\infty} R\left(\left(\lambda_{k}\right)\right)$, where $\lambda_{k}=\mu_{k}^{2}$.

By duality, we deduce that $G(\Delta)^{*}=C_{1}+R_{1}\left(\left(\lambda_{k}^{-1}\right)\right)$. More precisely, the operator space structure of $G(\Delta)^{*}$ is determined as follows: for any finite sequence $\left(x_{k}\right) \subset S_{1}$ :

$$
\|x\|_{S_{1}\left[G(\Delta)^{*}\right]}=\inf \left\{\left\|\left(\sum_{k} y_{k}^{*} y_{k}\right)^{1 / 2}\right\|_{S_{1}}+\left\|\left(\sum_{k} \lambda_{k}^{-1} z_{k} z_{k}^{*}\right)^{1 / 2}\right\|_{S_{1}}\right\}
$$

where the infimum runs over all decompositions $x_{k}=y_{k}+z_{k}$ in $S_{1}$. Therefore, by the Khintchine inequality in Theorem 6.13 with $p=1, G(\Delta)^{*}$ is completely isomorphic to $\mathcal{G}_{1}$ there. Thus we have proved the following

Theorem 7.3 $\mathrm{OH}$ is completely isomorphic to a subspace of a noncommutative $L_{1}$-space.

Remark 7.4 i) The proof of Theorem 7.3 gives much more. In fact, it shows that the dual space of any graph in $C \oplus R$ completely embeds into an $L_{1}$. From this one can deduce that a quotient of a subspace of $C \oplus R$ completely embeds into an $L_{1}$. We refer to [J2], [P4] and [X1] for more information.

ii) The von Neumann algebra $\Gamma$ is not hyperfinite. $O H$ also completely embeds into the predual of a hyperfinite algebra (see [J3], [HM]).

\section{References}

[BKS] M. Bożejko, B. Kümmerer and R. Speicher. q-Gaussian processes: non-commutative and classical aspects. Comm. Math. Phys., 185:129-154, 1997.

[BS] M. Bożejko and R. Speicher. An example of a generalized Brownian motion. Comm. Math. Phys., 137:519-531, 1991.

[ER] Ed. Effros and Z-J. Ruan. Operator spaces. The Clarendon Press Oxford University Press, New York, 2000.

[HM] U. Haagerup and M. Musat. On the best constants in noncommutative Khintchine type inequalities. J. Funct. Anal., to appear.

[HP] U. Haagerup and G. Pisier. Bounded linear operators between $C^{*}$-algebras. Duke Math. J., 71:889-925, 1993. 
[Ha] F. Hansen. An operator inequality. Math. Ann., 246:249-250, 1979/80.

[J1] M. Junge. Doob's inequality for non-commutative martingales. J. Reine Angew. Math., 549:149-190, 2002.

[J2] M. Junge. Embedding of the operator space $O H$ and the logarithmic "little Grothendieck inequality'. Invent. Math., 161:225-286, 2005.

[J3] M. Junge. Operator spaces and Araki-Woods factors: a quantum probabilistic approach. IMRP Int. Math. Res. Pap., pages Art. ID 76978, 87, 2006.

[JO] M. Junge and T. Oikhberg. Unconditional basic sequences and homogeneous Hilbertian subspaces of non-commutative $L_{p}$ spaces. Indiana Univ. Math. J., 56:733-765, 2007.

[JX] M. Junge and Q. Xu. Noncommutative Burkholder/Rosenthal inequalities II: Applications. Isreal J. Math., to appear.

[Ka] J-P. Kahane. Some random series of functions. Cambridge University Press, Cambridge, second edition, 1985.

[Ko] H. Kosaki. Applications of the complex interpolation method to a von Neumann algebra: noncommutative $L^{p}$-spaces. J. Funct. Anal., 56:29-78, 1984.

[KR] R. V. Kadison and J. R. Ringrose. Fundamentals of the theory of operator algebras. Vol. II. Amer. Math. Soc., Providence, RI, 1997.

[Pa] V. Paulsen. Completely bounded maps and operator algebras, volume 78 of Cambridge Studies in Advanced Mathematics. Cambridge University Press, Cambridge, 2002.

[LPP] F. Lust-Piquard and G. Pisier. Noncommutative Khintchine and Paley inequalities. Ark. Mat., 29:241-260, 1991.

[P1] G. Pisier. The operator Hilbert space OH, complex interpolation and tensor norms. Mem. Amer. Math. Soc., 122(585):viii+103, 1996.

[P2] G. Pisier. Non-commutative vector valued $L_{p}$-spaces and completely $p$-summing maps. Astérisque, (247):vi+131, 1998.

[P3] G. Pisier. Introduction to operator space theory. Cambridge University Press, Cambridge, 2003.

[P4] G. Pisier. Completely bounded maps into certain Hilbertian operator spaces. Int. Math. Res. Not., (74):3983-4018, 2004.

[P5] G. Pisier. The operator Hilbert space $\mathrm{OH}$ and type III von Neumann algebras. Bull. London Math. Soc., 36:455-459, 2004.

[PS] G. Pisier and D. Shlyakhtenko. Grothendieck's theorem for operator spaces. Invent. Math., 150:185-217, 2002.

[PX] G. Pisier and Q. Xu. Non-commutative $L^{p}$-spaces. In Handbook of the geometry of Banach spaces, Vol. 2, pages 1459-1517. North-Holland, Amsterdam, 2003.

[R] Z-J. Ruan. Subspaces of $C^{*}$-algebras. J. Funct. Anal., 76:217-230, 1988.

[S] D. Shlyakhtenko. Free quasi-free states. Pacific J. Math., 177:329-368, 1997.

[T] M. Takesaki. Theory of operator algebras. I. Springer-Verlag, New York, 1979. 
[VDN] D. V. Voiculescu, K. J. Dykema and A. Nica. Free random variables. Amer. Math. Soc., Providence, RI, 1992.

[X1] Q. Xu. Embedding of $C_{q}$ and $R_{q}$ into noncommutative $L_{p}$-spaces, $1 \leq p<q \leq 2$. Math. Ann., 335:109-131, 2006.

[X2] Q. Xu. Operator-space Grothendieck inequalities for noncommutative $L_{p}$-spaces. Duke Math. J., 131:525-574, 2006. 